\title{
POLARIMETRY AND UNIFICATION OF LOW-REDSHIFT RADIO GALAXIES
}

\author{
Marshall H. Cohen ${ }^{1}$, Patrick M. Ogle ${ }^{1,2}$, Hien D. Tran ${ }^{3,4}$ \\ Robert W. Goodrich ${ }^{5}$, Joseph S. Miller ${ }^{6}$
}

\begin{abstract}
We have made high-quality measurements of the polarization spectra of $13 \mathrm{FR}$ II radio galaxies and taken polarization images for 11 of these with the Keck telescopes. Seven of the eight narrow-line radio galaxies (NLRG) are polarized, and six of the seven show prominent broad Balmer lines in polarized light. The broad lines are also weakly visible in total flux. Some of the NLRG show bipolar regions with roughly circumferential polarization vectors, revealing a large reflection nebula illuminated by a central source. Our observations powerfully support the hidden quasar hypothesis for some NLRG. According to this hypothesis, the continuum and broad lines are blocked by a dusty molecular torus, but can be seen by reflected, hence polarized, light. Classification as NLRG, broad-line radio galaxy (BLRG), or quasar therefore depends on orientation. However, not all objects fit into this unification scheme. Our sample is biased towards objects known in advance to be polarized, but the combination of our results with those of Hill, Goodrich, \& DePoy (1996) show that at least 6 out of a complete, volume and flux-limited sample of 9 FR II NLRG have broad lines, seen either in polarization or $\mathrm{P} \alpha$.

The BLRG in our sample range from 3C 382, which has a quasar-like spectrum, to the highly-reddened IRAS source FSC $2217+259$. This reddening sequence suggests a continuous transition from unobscured quasar to reddened BLRG to NLRG. Apparently the obscuring torus does not have a distinct edge. The BLRG have polarization images which are consistent with a point source broadened by seeing and diluted by starlight. We do not detect extended nebular or scattered emission, perhaps because it is swamped by the nuclear source. Our starlight-corrected BLRG spectra
\end{abstract}

\footnotetext{
${ }^{1}$ California Institute of Technology, Pasadena, CA 91125; mhc@astro.caltech.edu

${ }^{2}$ present address: Massachusetts Institute of Technology; pmo@space.mit.edu

${ }^{3}$ Institute of Geophysics and Planetary Physics, Lawrence Livermore National Laboratory, 7000 East Ave, P.O. Box 808, L413, Livermore, CA 94550

${ }^{4}$ present address: Johns Hopkins University, Baltimore, MD 21218; tran@adcam.pha.jhu.edu

${ }^{5}$ CARA/Keck Observatory, 65-1120 Mamalahoa Highway, Kamuela, HI 96743; goodrich@keck.hawaii.edu

${ }^{6} \mathrm{UCO} /$ Lick Observatory, University of California, Santa Cruz, CA 95064; miller@ucolick.org
} 
can be explained with a two-component model: a quasar viewed through dust, and quasar light scattered by dust. The direct flux is more reddened than the scattered flux, causing the polarization to rise steeply to the blue. Strong rotations of the electric vector position angle across $\mathrm{H} \alpha$ in 3C 227 and 3C 445 may be explained by systematic orbital motions in an equatorial broad-line region.

Subject headings: galaxies: active — galaxies: nuclei — polarization — quasars: general

\section{Introduction}

Active galaxies can be classified in many ways, according to spectrum, luminosity, and morphology, and the result is a confused taxonomy that only partly reflects the underlying physics. This situation has led to "unification theories" in which various categories are linked by orientation, evolution or a gradient in physical characteristics. (For reviews, see e.g. Lawrence 1987, Urry \& Padovani 1995). The one that concerns us in this paper connects Type 1 (broad-line) and Type 2 (narrow-line) objects by aspect. In this picture an active galactic nucleus (AGN) contains a bright continuum source and a broad-line region (BLR) surrounded by a dusty torus. When viewed from near the pole the center is visible and a Type 1 object is seen, whereas along the equator the view of the center is blocked by the torus, and a Type 2 object is seen. When the conditions are right the nuclear light (continuum and broad lines) escaping along the poles can be scattered and thus polarized, and seen by the observer.

Miller and his collaborators (Antonucci \& Miller 1985, Miller \& Goodrich 1990, Tran, Miller, \& Kay 1992), with the aid of spectropolarimetry, showed that many Seyfert 2 galaxies do contain a hidden BLR, and a detailed investigation of the Seyfert 2 NGC 1068 (Miller, Goodrich, \& Mathews 1991) showed that the scattered light is typical of a Seyfert 1 nucleus. Thus unification by aspect is proven for many Seyfert 1 and 2 galaxies. Heisler, Lumsden \& Bailey (1997) surveyed Seyfert 2's selected by FIR fluxes and colors, which supposedly are isotropic quantities, and showed that at least 7 of 16 objects contain a hidden BLR. Also, infrared observations that penetrate the dust and see the BLR have shown directly that some Type 2 Seyferts have broad line regions Veilleux, Goodrich, \& Hill 1997).

In this paper our interest centers on the powerful FR II radio galaxies (RG), and on radio-loud QSO's (quasars). Like Seyferts, the RG come in Types 1 and 2 (BLRG and NLRG, respectively), and there has been interest in seeing if they also can be unified by aspect. They have the further virtue of containing a radio jet that presumably marks the dynamical axis of the black hole and accretion disk, providing additional geometric information. Quasars and RG are obviously anisotropic at radio wavelengths, and the viewing aspect has long been a major factor in their

unification (e.g.. Readhead et al. 1978). The common deficit of observed ionizing photons relative 
to those seen by the line-emitting regions (e.g. Neugebauer et al. 1980, Binette, Fosbury, \& Parker 1993) shows that RG also must be anisotropic at optical and UV wavelengths. A statistical basis for unifying RG and quasars was provided by Barthel (1989), who used the opaque torus scenario developed for Seyferts. Barthel postulated that most FR II RG and quasars are similar, and contain a continuum source and BLR inside an opaque torus. Quasars are seen within about 45 degrees of the pole where there is a direct view into the center, and RG are seen at higher inclinations where the quasar is partially or entirely hidden. Blazars are seen when the line of sight (LOS) is close to the axis, and the rapid variability in flux and polarization due to variations in beamed synchrotron radiation can be seen.

Many observations have now shown that much of this scenario must be correct. Broad $\mathrm{H} \alpha$ has been seen in the polarized light from the NLRG 3C 234 (e.g. Tran, Cohen, \& Goodrich 1995, hereafter T95), 3C 321 (Young et al. 1996), and Cygnus A (Ogle et al. 1997, hereafter O97). In all three objects an estimate of luminosity is consistent with their being off-axis quasars. Imaging polarimetry shows that in some RG the polarized regions have the azimuthal symmetry of a reflection nebula, as expected from an extended scattering region illuminated by a point source di Serego Alighieri, Cimatti, \& Fosbury 1993, Draper, Scarrott, \& Tadhunter 1993, Cohen et al. 1996, O97, Tran et al. 1998). Also, as with Seyferts, broad IR lines have been seen in a number of NLRG. Hill, Goodrich, \& DePoy (1996; hereafter H96) found broad P $\alpha$ in 4 of 9 NLRG that they surveyed, indicating that some NLRG contain a BLR hidden by dust, and would be called quasars if seen from a dust-free direction.

Another aspect of unification is the "alignment effect", the tendency of galactic extended emission line regions (EELR) to lie along the radio axis (see McCarthy 1993 for a review). At least part of the explanation involves an AGN in which the radio axis, the dusty torus, and the photoionization cones are all roughly coaxial. This picture is generally confirmed with polarimetry, which shows that the reflection nebula is spatially coincident with the EELR. Jet-induced star formation might also contribute to the alignment effect (e.g. Best, Longair, \& Röttgering 1996).

In 1994 we started a program of spectropolarimetric observations of powerful radio galaxies, to study unification and the alignment effect, and to study the geometry and kinematics of the AGN. We have already reported on 8 objects: PKS 0116+082, 3C 234, FSC 10214+4724, 3C 265, 3C 277.2, 3C 324, 3C 343.1, and Cygnus A. (Cohen et al. 1997, T95, Goodrich et al. 1996, Cohen et al. 1996, Tran et al. 1998, O97) In this paper we give the combined results for the 13 objects for which $z \leq 0.3$; i.e.., those in which $\mathrm{H} \alpha$ is available, and discuss the polarimetry, unification, geometric effects, and the continuum radiation. In general we assume that the scattering is due to dust, which undoubtedly is present, at least near the nucleus, because of the observed extinction. The relative importance of electron and dust scattering is undetermined, since dust can mimic electrons in producing wavelength-independent scattering in the optical band.

The organization of this paper is as follows. In $\S 2$ we describe our sample of FR II galaxies, $\S 3$ gives some observational details, and $\S 4$ and $\S 5$ give a general view of the results. Section 
6 presents a model for the continuum radiation for BLRG. Details of individual objects are in $\S 7$ and $\S 8$. In $\S 9$ we discuss the rotations in $\theta$ (polarization position angle) observed in 3C 227 and $3 \mathrm{C}$ 445. Section 10 contains further discussions of direct vs scattered light, unification, and radio-optical connections. We choose $\mathrm{H}_{\mathrm{o}}=65 \mathrm{~km} \mathrm{sec}^{-1} \mathrm{Mpc}^{-1}$ and $q_{o}=0.05$ for all calculations. We shall make use of both the full Stokes parameters $(Q, U)$ and the normalized parameters $(q, u)$; the use of upper and lower case letters should minimize any confusion.

\section{The Sample}

We have been measuring the polarimetric properties of radio galaxies with the 10-m W.M. Keck telescopes, and define those in which $\mathrm{H} \alpha$ is visible as the "low-z sample". To this Keck group we add two objects observed with the 5-m Hale telescope at Palomar Observatory. The total sample, listed in Table 1, comprises 13 FR II RG with $z \leq 0.3-5$ BLRG and 8 NLRG. 3C 234 is often called a BLRG because broad $\mathrm{H} \alpha$ is readily seen in $\mathrm{F}_{\lambda}$, but we list it with the NLRG because the broad line is predominantly composed of scattered light (See $\S 4.1$ ).

Column 6 of Table 1 gives an estimate of an upper limit to the interstellar polarization (ISP) towards each source. This value is obtained by estimating the extinction towards each object from the maps prepared by Burstein \& Heiles (1982), and using the empirical formula $p_{\max }=0.09 \times E(B-V)$ (Serkowski, Mathewson, \& Ford 1975). Cygnus A is at low latitude and is discussed by Tadhunter, Scarrott, \& Rolph (1990); see also O97. The ISP effects are small in this case. Possible ISP effects in 3C 33, 3C 195, and FSC 2217+295 are discussed in $\S 8$ and $\S 9$. References to previous polarimetric work are in column 7 of Table 1.

Our sample is not an approximation to a flux-limited or volume-limited sample, as most of the objects were chosen because they were known to be polarized. 3C 105 and 3C 135 were observed because they are FR II RG that fitted into a slot in the observing schedule. 3C 357 was observed because its HST image (de Koff et al. 1996) shows an off-nuclear emission region that we thought might be polarimetrically interesting. We shall make use of work by $\mathrm{H} 96$ who observed $\mathrm{P} \alpha$ in a complete sample of 11 RG (see $\S 10.2$ ), 3 of which are also in our sample. This will give our results more generality than they would have by themselves.

\section{Observations}

The Keck observations were made with the polarimetry module (Goodrich, Cohen, \& Putney 1995, Cohen et al. 1997) in the Low-Resolution Imaging Spectroscope (Oke et al. 1995). The polarimeter contains a rotatable half-wave plate and a calcite beamsplitter. The Stokes parameters are measured by taking 4 exposures at waveplate position angles (PA) of $0^{\circ}, 45^{\circ}, 22.5^{\circ}$, and $67.5^{\circ}$, and combining the spectra with the standard self-calibration procedure (Cohen et al. 1997), using the computer reduction system VISTA. At each epoch we observed standard polarized stars and 
standard null stars as a check on the system and to establish the reference for PA determinations. The standard stars are taken from the lists of Schmidt, Elston, \& Lupie (1992) or have been referenced back to that list by our own measurements with the Keck polarimeter. The relative accuracy in PA determinations is about $0.5^{\circ}$. The PA calibration is discussed in detail by Ogle (1998).

For spectroscopy we used a grating with $300 \mathrm{~g} / \mathrm{mm}$, blazed at $5000 \AA$. This gives a dispersion of $\sim 2.5 \AA$ per pixel and a resolution of $\sim 10 \AA$. The wavelength coverage was typically $3900 \AA$ to $8900 \AA$. The slit was $1.0^{\prime \prime}$ wide, except where noted in Table 2 . We did not observe with an order-blocking filter. In most cases the spectra are flat or rise to the red, and errors due to second-order light are negligible. We did observe the flux standards with and without the order-blocking filter, as most of them are hot white dwarfs and the flux calibration would otherwise have been in error.

For imaging we replaced the grating by a mirror and inserted a B-band filter into the beam. The scale is $0.215^{\prime \prime}$ per pixel. The imaging polarimetry procedure is the same as for spectroscopy, with the simplification that there is no spectral extraction. When necessary for either spectroscopy or imaging we improved the $\mathrm{S} / \mathrm{N}$ by binning the fluxes rather than by averaging the Stokes parameters themselves, which can lead to bias (Clark et al. 1983). We typically took 15-minute exposures so that a full 4-exposure set took an hour plus readout time.

The field of view of the Keck polarimeter is about $32^{\prime \prime}$. This limits the useful length of the slit for spectroscopy, and for all observations it can make sky subtraction difficult, as some galaxies nearly fill the field. In such a case the apparent polarization $p$ may be higher than the value a larger field would have given; this is analogous to increasing $p$ for a nuclear source by narrowing the slit and allowing less diluting starlight into the the measurement.

At Palomar we used the double spectrograph (Oke \& Gunn 1982) to do spectropolarimetry, but not imaging polarimetry, on the 5-m Hale telescope. The polarimeter is similar to that used at Keck (Goodrich 1991). In the red camera the grating has $158 \mathrm{~g} / \mathrm{mm}$ and dispersion approximately $6 \AA$ per pixel, and in the blue camera the grating has $316 \mathrm{~g} / \mathrm{mm}$ giving approximately $2.1 \AA$ per pixel. Calibration and data reductions were done in a fashion similar to those used at Keck.

Table 2 gives the log of all the observations. The imaging observations on 1995 December 16 were compromised by poor seeing that ranged up to $1.8^{\prime \prime}$. We reobserved 3C 195 and 3C 234 on 1996 April 16 and only the later data are included in this paper. Four RG, PKS 0116+082, 3C 109, 3C 227 and FSC 2217+259, were observed only in December 1995, and those data are included here except for PKS 0116+082, which was presented in Cohen et al. (1997). The high and variable seeing compromises the angular resolution, but does not further affect the polarization observations. 


\section{Results: Spectropolarimetry}

We display the combined spectropolarimetry results in Figures 1 and 2, where the objects are arrayed, roughly, in order of decreasing visibility of the broad $\mathrm{H} \alpha$ line in $\mathrm{F}_{\lambda}$. In Figure 1 the left panels show the total flux $\mathrm{F}_{\lambda}$ and the right panels show the "polarized flux" defined as $p \times \mathrm{F}_{\lambda}$. This quantity is sometimes called the "Stokes Flux", and in simple cases it is proportional to the scattered flux. The spectra of $\mathrm{F}_{\lambda}$ and $p \times \mathrm{F}_{\lambda}$ are shown on a logarithmic scale. Note that corresponding plots in Figure 1 have the same logarithmic range, so that the relative enhancement of $\mathrm{H} \alpha$ can be more readily assessed. Figure $2 \mathrm{a}$ shows the fractional linear polarization $p$ and Figure $2 \mathrm{~b}$ shows the polarization position angle $\theta$. When the $\mathrm{S} / \mathrm{N}$ is high $(>5)$ we can estimate $p$ as $\sqrt{\left(q^{2}+u^{2}-\sigma^{2}\right)}$ where $q$ and $u$ are the normalized Stokes parameters and $\sigma$ is the error in $q$ or $u$ calculated from photon statistics and the normal propagation of errors (Simmons \& Stewart 1985). In most cases, however, $\mathrm{S} / \mathrm{N}$ is not that high, especially at the blue end of the spectrum. Instead of attempting to debias the square-root, we prefer to rotate $q$ and $u$ by $-2 \Theta$, where $\Theta$ is a smooth approximation to the measured $\theta$ (Cohen et al. 1997). The resulting $q^{\prime}$ is a useful estimate of $p$. Hereafter we use the symbol $p$ for all estimates of the polarization.

In Figures 1 and 2 we show spectra for 3C 109, 3C 234 and Cyg A that have been published before (Goodrich \& Cohen 1992, hereafter G92; T95, O97) and are repeated here to give a more complete impression of the range of possible behaviors. 3C 105 had no measurable polarization and spectra for it are shown in $\S 8.3$. The polarization of $3 \mathrm{C} 357$ is particularly noisy and it is omitted from Figures 1 and 2 but is shown in Figure 3 and discussed in $§ 8.7$. Generally the spectra are for a region centered on the nucleus, except for $3 \mathrm{C} 33$ where the extraction window was centered on a strongly-polarized region approximately $3^{\prime \prime}$ north-east of the nucleus, and $3 \mathrm{C}$ 357 where the window is approximately $1.5^{\prime \prime}$ west of the nucleus. The length of the window is given in Table 3, which gives some results from the polarimetry. The averaging interval, 5400 - $5600 \AA$, contains no strong emission lines, so the values of $p$ and $\theta$ represent, approximately, the V-band continuum polarization. However, we show in $\S 5.2$ that many of the NLRG contain reflection nebulae, in which $\theta$ varies with position. In these objects the measured $\theta$ may depend on the location of the slit and the extraction window.

For most objects an elliptical galaxy template was fit to the absorption features and subtracted from $\mathrm{F}_{\lambda}$ to get the "corrected flux" $\mathrm{F}_{\lambda, c}$. The corrected polarization $p_{c}=p /\left(1-f_{g}\right)$ is in Table 3. We used NGC 6702 as the template galaxy except for 3C 234 and Cyg A where NGC 821 was used. Two BLRG, 3C 382 and 3C 445, have so little starlight that we were unable to determine an accurate galaxy component other than $f_{g}<0.1$. We were unable to fit 3C 109 properly, but the absorption lines are weak and the galaxy fraction must be small. Figure 3 shows curves of $\mathrm{F}_{\lambda, \mathrm{c}}$ and $p_{\mathrm{c}}$ for 7 objects; those for 3C 234 and Cygnus A have already been published (T95, O97).

At this point we discuss the main features of Figures 1-3. Details will be presented in later sections. 


\subsection{Broad vs Narrow Line Objects and 3C 234}

It has been customary to define a broad-line radio galaxy merely as one that has prominent broad lines, e.g. the Balmer series, that are substantially broader than the forbidden lines e.g. [OIII]. This simple definition, however, takes no account of equivalent widths, and is subject to the vagaries of observation and signal-to-noise ratio. Further, it does not allow for differing geometric or physical circumstances. In Figure 1a, 3C 234 shows prominent broad $\mathrm{H} \alpha$ and easily seen broad $\mathrm{H} \beta$. These lines were noted long ago and thus 3C 234 is usually called a BLRG Grandi \& Osterbrock 1978). Note, however, that the broad lines are noticeably weaker than those in the top 5 galaxies in Figure 1a, and 3C 234 has also been called an NLRG. Antonucci \& Barvainis (1990) argue by analogy with the Type 2 Seyfert NGC 1068 that the broad lines are polarized like the continuum and are predominantly due to scattered light, and that 3C 234 should be called a Type 2 object (see also T95). We agree that the broad lines are mainly due to scattering, because broad $\mathrm{H} \alpha$ and $\mathrm{H} \beta$ are more prominent in $p \times \mathrm{F}_{\lambda}$ than in $\mathrm{F}_{\lambda}$. Thus we discuss $3 \mathrm{C} 234$ with the NLRG. However, it is intermediate between Type 1 and Type 2 objects, and has other differences from the BLRG and NLRG in addition to the intermediate equivalent width of the broad lines. We discuss this in $\S 8.5$.

\subsection{Continuum Radiation}

In Figure 1a the BLRG have been arrayed in order of decreasing visibility of broad $\mathrm{H} \alpha$, but it can be seen that the order is also one of increasing redness and $p$. 3C 382 has a blue quasar spectrum and is weakly polarized; FSC $2217+259$ is a red IRAS galaxy and is highly polarized. The correlations are shown in Figure 7, along with calculations made with a simple model which is presented in $\S 6$. Briefly, the model assumes that the BLRG continuum (after starlight subtraction) is a combination of two components, a nearly unpolarized direct ray that has a view of the nuclear continuum source and BLR but has extinction and reddening due to dust, and a highly polarized scattered ray that has less reddening. The net polarization increases as the ratio of scattered to direct radiation increases, so the direct ray bluens the polarization and reddens the total flux.

This model is similar to the one used by Wills et al. (1992) for IRAS 13349+2438. Application to individual objects is given in $\S 7$.

The continuum model cannot be used for the NLRG because they have an additional important component of radiation. Most of the NLRG, along with many Seyfert 2 galaxies, have broad lines that are more highly polarized than the neighboring continuum, even after the subtraction of starlight (Figure 3b). This led Tran (1995) to consider two featureless continuum components; the first, FC1, is due to scattering and is polarized, while the other, FC2, is assumed to be unpolarized. FC2 differentially dilutes the continuum and $\mathrm{H} \alpha$; this leaves $p_{\mathrm{c}}(\mathrm{H} \alpha)>p_{\mathrm{c}}(\mathrm{cont})$. The relative levels of FC1 and FC2 can be estimated by requiring the broad $\mathrm{H} \alpha$ emission line to have the same intrinsic polarization as the neighboring FC1. This is useful if it can plausibly be 
maintained that the AGN light (continuum and broad lines) is all polarized at the same level, by scattering. We think that this is a conservative assumption, as it is unlikely that the broad lines could be intrinsically more polarized than the nuclear continuum. They surely come from a larger region (Tran 1995), and are more likely to be intrinsically polarized less than the continuum, as in 3C 382 (see also Martel 1996, Ogle et al. 1999).

In 3C 234 the decomposition into FC1 and FC2 (T95) showed that the continuum may consist of three components in addition to unpolarized starlight: Balmer nebular continuum radiation from the NLR, nuclear light in which the continuum and broad lines are polarized the same (FC1), and an FC2 whose origin is unclear. The reliability of this model was enhanced when it turned out that broad $\mathrm{H} \beta$ had the same $p$ as its neighboring $\mathrm{FC}$, even though $\mathrm{H} \beta$ was not used in the construction. Cygnus A yields a similar result (O97), and in this case FC2 can be interpreted as diluting radiation from hot young stars Goodrich \& Miller 1989; see also Heckmann et al. 1995, Storchi-Bergmann, Fernandes, \& Schmitt 1998).

\section{3. $\quad p$ and $\theta$}

Nearly every object has $p$ rising to the blue (Figure 2a). For most objects this is mainly due to the reduction of dilution of $p$ by unpolarized light from an old stellar population. (This point has been made numerous times; see e.g. T95, and Cimatti \& di Serego Alighieri 1995). It is generally said that most low-z RG have low $p$ whereas most high-z RG have high $p$, and that this is due to the different rest-wavelength ranges covered at low and high z. Figure 2 shows this wavelength dependence: at $5500 \AA$ all but 3C 109, FSC $2217+259$ and 3 C234 have $p<3 \%$, whereas below $4000 \AA$ all but 3C 382, 3C 445 and 3C 135 have $p>3 \%$. However, Figure 3b shows that $p_{\mathrm{c}}$ still rises to the blue in most objects. As remarked above, this could be caused by another diluting component; e.g. a reddened direct ray or FC2.

The rise of $p_{\mathrm{c}}$ to the blue, especially in the UV, means that the polarized flux $p \times \mathrm{F}_{\lambda}$ has a steeper spectrum than the galaxy-corrected flux $\mathrm{F}_{\lambda, \mathrm{c}}$. In $p \times \mathrm{F}_{\lambda} 3 \mathrm{C} 382$ and 3C 321 both have an index $\alpha \approx-2.0\left(\mathrm{~F}_{\nu} \sim \nu^{-\alpha}\right)$, at least blueward of $\mathrm{H} \alpha$. This is part of the evidence that $3 \mathrm{C} 321$, an NLRG, has a quasar-like central continuum source. See $\S 8.6$.

The position angle $\theta$ (Figure $2 \mathrm{~b}$ ) is remarkably constant in many of the objects. In FSC $2217+259$, for example, a linear fit to $\theta$ gives a slope $0.0004^{\circ} \pm 0.0003^{\circ} / \AA$ which is at most $2^{\circ}$ across the spectrum. In $3 \mathrm{C} 234$ a similar fit gives a change $\Delta \theta=-2.5^{\circ} \pm 4.4^{\circ}$ across the spectrum. In these objects there can be little polarization by intervening dust extinction, either in our galaxy or the host galaxy, because $p$ itself changes strongly with wavelength and the observed $\theta$ would rotate noticeably, unless there were a fortuitous alignment of position angles. A few objects do have a significant rotation in the position angle of the continuum. The two objects with double nuclei have large rotations; 3C 321 rotates $\sim 28^{\circ}$ between $3550 \AA$ and $7000 \AA$, and Cygnus A has $\Delta \theta=19^{\circ} \pm 4^{\circ}$. The Cygnus A rotation is explained with three components of continuum 
radiation, with different $p$ 's and $\theta$ 's, that combine to give the observed values (O97).

\subsection{Polarization of the Broad Lines}

In all the polarized NLRG except 3C 357, $p$ rises in broad $\mathrm{H} \alpha$ (Figure 2a). This is due to differential dilution by starlight and other components. It is this increase in $p$ that makes broad $\mathrm{H} \alpha$ more prominent in the polarized flux $p \times F_{\lambda}$ than in $F_{\lambda}$ (Figure 1 ). 3C 357W is the only

polarized object that does not show broad lines. However, this object is faint and the telluric A band falls on the red wing of $\mathrm{H} \alpha$, so this result is inconclusive (see $\S 8.7$ ). 3C 382 is different from the other BLRG as it has a quasar spectrum, with a weakly polarized continuum and little or no starlight or narrow lines. Broad $\mathrm{H} \alpha$ and $\mathrm{H} \beta$ are weak in $p \times F_{\lambda}$, and are nearly unpolarized. Weak polarization of the broad lines relative to the continuum is typical of quasars (Ogle et al. 1999).

The sequence in Figure $1 \mathrm{~b}$ shows that broad $\mathrm{H} \alpha$ is similar in polarized flux from 3C 227 to at least $3 \mathrm{C} 33$. In these cases the polarized flux is giving us a look deep into the interior of the object, by scattering. We also see the interior of the BLRG directly, but with extinction (see $\S 6$ ). This may be true also for 3C 234 (T95). In 3C 234, and in some of the BLRG, the LOS is probably near the edge of the obscuring torus. We suspect that the torus does not have a sharp boundary, and that there is a range of inclinations where the interior can be seen through dust.

\subsection{Narrow emission lines}

In general the narrow lines are only weakly polarized. This is readily seen in Figure 2a, where many objects show strong reductions in $p$ at the locations of the narrow lines. This is most striking in 3C 234 where more than a dozen narrow lines show the effect strongly. Narrow $\mathrm{H} \alpha$ shows up prominently as a reduction in $p$ in 3C 234, 3C 33NE, and 3C 195 because these are the objects that are dominated by narrow lines in total flux but by broad lines in polarized flux.

All the objects except 3C 109, 3C 234, and 3C 382 show narrow emission lines in $p \times F_{\lambda}$ (Figure 1b), so these lines are polarized but at a low level. The possible polarization of the [O III] lines in 3C 109 is discussed in G92. Weakly polarized [O III] $\lambda \lambda 4959,5007$ is seen in most objects, several objects show polarized narrow $\mathrm{H} \alpha$, and Cyg A shows several other species. [O II] $] \lambda 3727$ is not reliably identified in any of the $p \times F_{\lambda}$ spectra, and is essentially unpolarized. The origin of [O II] must be at or outside the scattering region, whereas the other narrow lines arise from deeper locations and are partly seen by scattering. The possible anisotropy of [O II] and [O III] and its interpretation in terms of unified AGN models has been discussed by e.g. Jackson \& Browne (1990), Hes, Barthel \& Fosbury (1993), Baker (1997), and di Serego Alighieri et al. (1997). 


\section{Results: Imaging Polarimetry}

We took polarimetric images of 11 objects (Table 2). Earlier observations of this type are referenced in Table 1. Our current work shows that the polarization images fall into two main classes, those with compact and those with extended polarized regions. There is a strong correlation between this image class and the spectral classification: the BLRG are compact and the NLRG are extended.

\subsection{BLRG}

The compact systems are shown in Figure 4. The innermost contour is $50 \%$ of the peak for every object, and except for FSC $2217+259$ the next contour is $5 \%$ of the peak (see the caption). All the objects except FSC $2217+259$ have FWHM $\approx 1^{\prime \prime}$, consistent with seeing. 3C 109 and 3C 227 have been called " $\mathrm{N}$ " galaxies (Spinrad et al. 1985), indicating that they contain a brilliant point-like nucleus. Other N galaxies in our sample include 3C 135 and 3C 234, shown in Figures 5 and 6 , and $3 \mathrm{C} 445$, for which we have no imaging data. 3C 382 has a strong nucleus although apparently it was not called an $\mathrm{N}$ system. In $\S 7.4$ we give evidence that it was exceptionally bright at our epoch.

3C 382, 3C 109, and 3C 227 are closely similar. The galaxies are dominated by a bright point source at the nucleus, and the polarization patterns are simple. The vectors are roughly parallel and decrease radially by a small amount - a factor of 0.8 in 3C 109 and 3C 382, and 0.6 in 3C 227. These patterns can be explained with a polarized point source broadened by seeing and diluted by galaxian starlight and by spatial averaging. There is no need to invoke scattering by an extended distribution of dust or electrons to explain the observations. FSC $2217+259$ is highly polarized and consequently has high $\mathrm{S} / \mathrm{N}$. The vectors are accurately parallel and decrease radially by a factor of two or three. It is similar to the other BLRG and the polarization pattern could again be due to seeing plus dilution, although the angular extent is greater than for the others and scattering in an extended medium may also play a role. This object is very red and we are seeing the nucleus through dust, as discussed in $§ 7.4$.

In 3C $382 p(\mathrm{H} \alpha)<p($ cont) and the scattering region is largely interior to the BLR. It may not be coincidental that 3C 382 has the weakest polarization of the BLRG. In 3C 109 and FSC $2217+259 p(\mathrm{H} \alpha)=p$ (cont) and $\theta$ does not change across $\mathrm{H} \alpha$; the main scattering region must be well outside the BLR. In 3C 227 and 3C $445 \theta$ rotates in $\mathrm{H} \alpha$; this is discussed in $\S 9$.

\subsection{NLRG}

Images for the NLRG are shown in Figures 5 and 6, except for Cygnus A, which has been extensively discussed elsewhere (O97). In Figure 5 the top panels show the total flux, the 
polarization vectors, and the radio axis. The lower panels show contours of polarized flux $p \times \mathrm{F}_{\lambda}$, with the slit location. Figure 6 shows total flux and polarization vectors for the two NLRG that have weak polarization and do not have extended polarization regions. In $3 \mathrm{C} 33,3 \mathrm{C} 195$, 3C 321, and Cyg A (O97) the polarized regions are in "fans" on opposite sides of the nucleus, and the polarization vectors are roughly azimuthal; i.e. they are tangential to circles around the center. This is the sign of a bipolar reflection nebula. A central source illuminates material in a bicone, and the scattered light is polarized perpendicular to the plane of scattering. Although the perpendiculars to the polarization vectors accurately define a center when averaged, seeing plus spatial averaging tilt the individual vectors away from perpendicular.

In all the objects with polarization fans the polarization is weak at the nucleus, and has a strong radial gradient. The sign of the gradient is opposite to that for the BLRG, where $p$ decreases with radius. The gradient must be partly due to dilution by unpolarized starlight, which is more strongly concentrated to the nucleus than is the scattering material. However, the effect is also seen in $3 \mathrm{C} 265$ with $\mathrm{z}=0.811$ (Tran et al. 1998). In this case the polarization image was measured in the rest-frame UV, and dilution by an old stellar population would have had little importance. Seeing and spatial averaging also affect the pattern, but they generally act to reduce the gradient.

Three of the four objects in Figure 5 and also Cyg A (O97); i.e. all the strongly polarized NLRG except for 3C 234, have separated peaks in $p \times \mathrm{F}_{\lambda}$. Since starlight is unpolarized it has no effect on $p \times \mathrm{F}_{\lambda}$ and we must look elsewhere for the cause. We suggest it is due to the presumed geometry, an illuminated cone whose apex is shielded by an opaque torus. Near the apex the resolution element is larger than the cone and the vector sum of the polarizations is reduced because they have a variety of angles, all perpendicular to the radius. The central decrease could also be caused by obscuration of the polarized light.

3C 234 is special. The polarization pattern is extended but does not show fans, and $p$ has a steady EW gradient with a peak east of the flux peak. $p \times \mathrm{F}_{\lambda}$ has a maximum at the center. This pattern is different from both the BLRG and the NLRG. The vectors show a tendency for circular symmetry, although not as strongly as the other objects, and the pattern may be due to scattering of nuclear light. This is discussed further in $\S 8.5$.

\section{BLRG Spectral Index, Polarization and Reddening}

In this section we first discuss the spectral index and Balmer decrement for the BLRG in terms of one reddening screen, and then present a two-component model that also attempts to explain the polarization. In $\S 7$ the results are applied to the individual sources. 


\subsection{One-Component Model - Absorption Only}

Assume that there is a dust screen in front of the BLRG which produces the same extinction in the continuum and the lines. Then we have two measures of reddening: the ratio of broad $\mathrm{H} \alpha$ to broad $\mathrm{H} \beta$, and the ratio of the continuum fluxes at $\mathrm{H} \alpha$ and $\mathrm{H} \beta$. The data plotted in Figure 7a show that these quantities are correlated. They have been corrected for Galactic reddening, and 3C 227, 3C 234, and FSC 2217+259 have been corrected for an old stellar population (the other objects have $f_{g}<0.1$ ). The continuum spectral index $\alpha$ between $\mathrm{H} \alpha$ and $\mathrm{H} \beta$ is shown in the top scale. In Fig 7 the order of the objects (left to right) is the same as the vertical order in Figure 1 except that Galactic dereddening has moved 3C 109 to the left of 3C 445. Baker (1997, Fig. 16) shows a similar diagram for radio-loud quasars. The range of Balmer decrement in Figure 7a is about the same as in Baker, but FSC $2217+259$ has a considerably redder spectrum than any of her quasars.

Four of the five BLRG (excluding 3C 109) form a sequence that is parametric in reddening. We model this relationship by assuming that the intrinsic Balmer decrement of the broad-line clouds is 3.0 (Case B). The reddening line for this model depends on the intrinsic spectral index $\alpha_{\mathrm{o}}$ of the continuum source, and in Figure 7a we plot solid lines for $\alpha_{\mathrm{o}}=-0.5,0.0,0.5$, and 1.0. The great majority of quasar indices fall within this range (Francis et al. 1991, Baker \& Hunstead 1995). The dotted lines show extinction $A_{V}$ in magnitudes, calculated with the Cardelli, Clayton, \& Mathis (1989) extinction law with $R_{V}=3.1$.

The model can be adapted to all the BLRG except 3C 109. However, radio loud quasars (excluding CSS sources) appear to have dereddened intrinsic indices close to 0.5 Baker \& Hunstead 1995), and FSC 2217+259 lies rather far from that value. It may be that it has an exceptional index, but it may also be that the model is too simple in assuming that the extinction is the same for both the continuum and broad lines. If the extinction to the BLR were more than to the continuum source, then FSC $2217+259$ could be accommodated with an index near 0.5. Indeed, the extra extinction could be associated with the BLR itself. For a discussion of dust in the BLR see e.g. Goodrich (1995). Another alternative is that the intrinsic Balmer decrement is substantially higher than 3.0 Kwan \& Krolik 1981).

3C 109 is well outside the range of the model. It is exceptional, either in the intrinsic Balmer decrement or the intrinsic index (or both). Alternatively, the model may not apply to 3C 109, at

least not in this simple form. 3C 234 is shown for comparison only; the model cannot be used for the NLRG because the scattered light arises far from the nucleus and cannot be presumed to be behind the nuclear screen. Also, the NLRG show an important radiation component, FC2, that is not accounted for here. 


\subsection{Two-Component Model}

Figure $7 \mathrm{~b}$ shows the galaxy-corrected continuum polarization at $5500 \AA$ (rest) vs $\mathrm{F}_{\lambda, \mathrm{c}}(\mathrm{H} \alpha) / \mathrm{F}_{\lambda, \mathrm{c}}(\mathrm{H} \beta)$ for the five BLRG and 3C 234. There is a trend for the polarization to increase with reddening. Rudy et al. (1983) made a similar plot for 13 BLRG; their sample includes all of our objects except FSC $2217+259$. They explained the correlation between $p$ and the Balmer decrement as either dichroic absorption or scattering with extinction. The hidden nucleus model for NLRG leads us to consider a more elaborate model to explain the correlation. It consists of two components: an unpolarized direct ray, and a ray from the nucleus that is scattered and highly polarized. The components both have extinction, but the direct ray has more. This is consistent with the presumed BLRG geometry in which we are near the edge of the cone. The direct ray must penetrate more dust than the scattered ray that passes closer to the axis. The effect of the model is to redden the continuum, increase the Balmer decrement, and bluen the polarization by differential dilution.

The model is similar to that used by Wills et al. (1992; see also Brotherton et al. 1998), except we assume that the extinction of the scattered component arises from dust inside the scattering volume itself. This has the advantage that the scattering and extinction are coupled, and there is one less free parameter. The direct ray can be expressed as $\mathrm{F}_{\mathrm{d}}=\mathrm{F}_{\mathrm{o}} \mathrm{e}^{-\tau_{\mathrm{d}}}$, where $\mathrm{F}_{\mathrm{o}}$ is the unobstructed flux from the nucleus, and $\tau_{\mathrm{d}}$ is the optical depth. To properly describe the scattered ray we should specify a geometry and integrate through the scattering volume. Such calculations have been done by Manzini \& di Serego Alighieri (1996) for a bicone uniformly filled with dust, but we merely make the simple approximation used by Fosbury et al. (1999). We assume that the scattered light can be expressed (approximately) as $\mathrm{F}_{\mathrm{s}}=\mathrm{F}_{\mathrm{o}} \mathrm{k}_{\mathrm{g}} \mathrm{Q}_{\mathrm{s}} \mathrm{e}^{-\tau_{\text {ext }}}$, where $\mathrm{k}_{\mathrm{g}}$ is a geometric factor and $\mathrm{Q}_{\mathrm{S}}$ is a scattering efficiency that depends on the size distribution of the dust particles and on their composition. Each ray passes through a dusty region before and after it is scattered, and the factor $\mathrm{e}^{-\tau_{\mathrm{ext}}}$ accounts for the extinction in the scattering volume. The

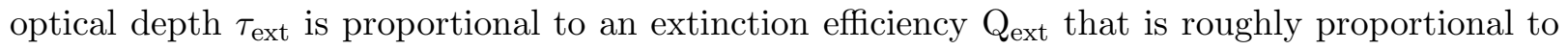
the scattering efficiency $Q_{s}$, provided the wavelength is not near the graphite resonance at 2200 $\AA$. The net result of these assumptions is that the scattered ray can be expressed as

$$
\mathrm{F}_{\mathrm{s}}=\mathrm{F}_{\mathrm{o}} \mathrm{k}_{\mathrm{s}} \tau_{\text {ext }} \mathrm{e}^{-\tau_{\mathrm{ext}}}
$$

The function $\tau_{\text {ext }} e^{-\tau_{\text {ext }}}$ has a maximum at $\tau_{\text {ext }}=1$, and most of the contributions to $\mathrm{F}_{\mathrm{s}}$ come from regions near $\tau_{\text {ext }} \approx 1$. For simplicity we now set $\tau_{\text {ext }}=1$ and Eq (1) becomes

$$
\mathrm{F}_{\mathrm{s}}=0.37 \mathrm{k}_{\mathrm{s}} \mathrm{F}_{\mathrm{o}}
$$

where $\mathrm{k}_{\mathrm{s}}$ is independent of wavelength except for geometric factors associated with the change of

the $\tau_{\text {ext }}=1$ surface with wavelength. $\mathrm{k}_{\mathrm{s}}$ is an adjustable parameter; we expect $\mathrm{k}_{\mathrm{s}}<<1$ and in the models shown below we use $\mathrm{k}_{\mathrm{s}}=0.05$. 
The scattered flux $\mathrm{F}_{\mathrm{s}}$ can be highly polarized; the curves by Manzini \& di Serego Alighieri (1996) show that the intrinsic polarization on $\mathrm{F}_{\mathrm{s}}, p_{\mathrm{s}}$, can be as high as $50 \%$. The apparent polarization $p_{\mathrm{c}}$ is

$$
p_{\mathrm{c}}=p_{\mathrm{s}} \mathrm{F}_{\mathrm{s}} /\left(\mathrm{F}_{\mathrm{s}}+\mathrm{F}_{\mathrm{d}}\right)
$$

Equation (2) states that the scattered flux is proportional to the incident flux; what we have done is to crudely justify the common assumption that the intrinsic polarization is independent of wavelength. The calculations by Manzini \& di Serego Alighieri, however, show that $p_{\mathrm{s}}$ generally rises to the red.

The model can be parameterized in various ways. In Figure $7 \mathrm{~b}$ we show lines of constant $\alpha_{\mathrm{o}}$ (solid), and horizontal lines of constant extinction for the direct ray, $A_{V}(d)$ (dotted), together with the corrected data. In this example the fixed parameters are $\mathrm{k}_{\mathrm{s}}=0.05$ and $p_{\mathrm{s}}=0.3$. The model also predicts the Balmer decrement and this is shown in Figure 7c, which is analogous to Figure 7a. The Balmer decrement and the index are limited because $\tau_{\text {ext }}=1$, and as $A_{V}(d)$ increases beyond 3 mag the scattered ray becomes dominant.

3C 109 and FSC 2217+259 are outside the range of the model in Figure 7c, and they are at the extreme values of $\alpha_{\mathrm{o}}$ in Figure $7 \mathrm{~b}$. The BLRG of course must have a range in their intrinsic properties, but it is difficult to fit 3C 109 and FSC 2217+259 into the scheme described here. Enlarging the model by adding excess extinction to or in the BLR is one way to accommodate them. Another is by postulating the existence of an additional blue continuum component. In both cases it must be done in a way that preserves the near equality of $p_{\mathrm{c}}$ for $\mathrm{H} \alpha$ and the continuum; i.e. the equivalent widths of the broad lines must be similar for all the rays. In studying the reddening of radio-loud quasars, Baker (1997) found analogous discrepancies. She suggests that excess reddening to the BLR could be the explanation, but that other factors could also be responsible. Optical depth effects that increase the intrinsic Balmer decrement could be part of the answer, as could dichroic dust extinction. See $\S 7.1$.

The point to this exercise is to show that the simple two-component model is able to explain qualitatively the observed spectra and polarimetry of the BLRG. Further insight could be obtained with detailed calculations and a specific geometric model, and by examining the full spectrum rather than just the two-point spectral index.

\section{Individual Objects: BLRG}

\section{1. $3 \mathrm{C} 109$}

The Palomar spectropolarimetry of 3C 109 is discussed by G92 who interpreted the polarization as due to transmission through aligned dust in the host galaxy. In view of the success 
of the two-component model, we now think that the origin of the polarization in 3C 109 should be reinvestigated. 3C 109 shows a Serkowski-type curve of $p(\lambda)$ (Figure 2a), but the data are noisy below $5000 \AA$. The appropriate way to discriminate between scattering and dichroic dust extinction is by extending the spectropolarimetry into the UV.

In Figures 7a and 7c 3C 109 stands apart from the other BLRG. We can describe this by saying that the Balmer decrement is higher than expected from the continuum slope. This could result from an intrinsically high Balmer decrement, or from extra reddening to the BLR. Alternatively, we could say that the continuum slope is bluer than expected from the Balmer decrement, and this could result from an extra blue continuum component that is not in the model. Evidently there is a number of possibilities for explaining the discrepancy; and, in addition, there remains the possibility that dichroic extinction plays an role.

\section{2. $\quad 3 \mathrm{C} 227$}

Prieto et al. (1993) have done extensive modeling of the EELR in 3C 227. They show that the velocity field can be modeled (roughly) with a bicone of opening angle $120^{\circ}$, tilted $40^{\circ}$ to the plane of the sky, and rotating about an axis offset $20^{\circ}$ from the cone axis. The projected bicone axis is at $\mathrm{PA}=40^{\circ}$ and the projected rotation axis is at $\mathrm{PA}=60^{\circ}$. We look inside a cone but near the edge. Their analysis of the emission lines shows that there is a photon deficit, and they suggest that 3C 227 harbors a quasar. Although the broad lines are prominent the quasar is "hidden" because most of the ionizing radiation is not directly visible.

The optical continuum radiation is polarized at $41^{\circ}$, which is parallel to the bicone axis. This is perpendicular to the orientation expected from scattering in the cone, but is consistent with the orientation expected from an optically thin disk coaxial with the cone. The radio axis is at $85^{\circ}$, which does not seem to be closely related to any of the above angles. The broad lines are polarized at $\sim 20^{\circ}$ (see $\S 9$ ) which is $20^{\circ}$ (in projection) from the cone and continuum radiation axes. $\theta$ shows notable rotations in the broad Balmer lines; we discuss them in $§ 9$. The rotation in $\mathrm{H} \alpha$ has already been noted by Corbett et al. (1998).

The spectrum of $3 \mathrm{C} 227$ can be qualitatively understood with the one-component model in $\S 6$. From Figure 7a, the Balmer decrement implies 1.1 magnitudes of extinction, and the color is then explained with $\alpha_{\mathrm{o}}=0.5$, the typical value for radio-loud quasars. To also explain the polarization, especially the fact that $p_{\mathrm{c}}$ rises to the blue, we need to use the two-component model. Figure $7 \mathrm{~b}$ shows that $\alpha_{\mathrm{o}} \approx-0.2$ and $\mathrm{A}_{\mathrm{V}}(\mathrm{d}) \approx 1.7$. These particular values can be modified by varying the other parameters in the model, but $\alpha_{\mathrm{o}}$ remains somewhat low. With $\mathrm{A}_{\mathrm{V}}(\mathrm{d})=1.7 \mathrm{mag}, p_{\mathrm{o}}=0.3$ and $\mathrm{k}_{\mathrm{s}}=0.05$ the calculated apparent polarization rises from $1.7 \%$ at $7000 \AA$ to $4.6 \%$ at $4000 \AA$. This rise is steeper than shown in Figure $3 \mathrm{~b}$, but the result is qualitatively correct. To use the model to match the polarization, $A_{V}$ would have to be reduced to near 1 mag, and the product $\mathrm{k}_{\mathrm{s}} p_{\mathrm{o}}$ would have to be doubled. 
The point-source polarization image in Figure 4 supports the scenario of Prieto et al. (1993) described above, in which we are looking into the cone but near the edge. Their photon deficit is

"... about a factor 2 for regions closer than $50 \mathrm{kpc}$, and a factor 13 for those located at much larger distances." Our estimate of $1-2$ mag for the V-band extinction to the nucleus is in rough agreement with their estimate of the photon deficit. They conclude that the intrinsic luminosity of $3 \mathrm{C} 227$ could put it in the quasar class.

\section{3. $\quad 3 \mathrm{C} 382$}

\subsubsection{Aperture Photometry}

Our imaging observations of 3C 382 were saturated in three of the four exposures in the extraordinary beam of the polarimeter, even though we reduced the exposure substantially from the expected value. They were not saturated in the ordinary beam because the throughputs of the two sides differ by about $5 \%$. And, as seen in Figure 4, the image shows the hexagonal diffraction pattern due to the secondary support and the mirror shape. Clearly, 3C 382 contained an unusually bright point-like nucleus during our observations. That night we also observed the standard stars PG 1525-071A and B (Landolt 1992) and used them as references. The 2 stars were self-consistent to better than 0.01 mag. We measured fluxes within a 2.1" aperture. The stars were observed about 4 hours before 3C 382. Two other radio galaxies were observed between the stars and $3 \mathrm{C} 382$, and each had 4 exposures that show variations of 0.05 mag. The night, however, was not photometric. With this caveat we find $\mathrm{B} \approx 14.5$ and $\mathrm{M}_{\mathrm{B}} \approx-23.0$ (allowing $0.3 \mathrm{mag}$ for Galactic extinction), for the night of 16 April 1996 (UT).

\subsubsection{Spectroscopy}

3C 382 shows double peaks in $\mathrm{H} \alpha$; this has been interpreted in terms of a rotating disk (Eracleous \& Halpern 1994). We made the spectropolarimetric observations on 19 May 1996 (UT). Flux calibrations were performed with HZ 44, but the night was not photometric. Figure 1 shows the observed spectrum. Within our sample, 3C 382 is exceptional in several respects. $\mathrm{F}_{\lambda}$ rises strongly to the blue, the narrow [O III] lines are very weak, and the broad lines are almost as wide (at least 26,000 km/sec FWZI) as the widest found in quasars (Kinney et al. 1991).

We can compare our flux values with those of Corbett et al. (1998) who observed the $\mathrm{H} \alpha$ region of $3 \mathrm{C} 382$ with a $1^{\prime \prime}$ slit on $6 / 7$ June 1995 . Their value for the continuum flux at $6500 \AA$ (observed) is about $7 \mathrm{mJy}$ (Figure 1 in Corbett et al.). Our value is $30 \mathrm{mJy}$. Corbett's value for the total flux at the peak of $\mathrm{H} \alpha$ (actually, the valley between the two peaks) is about $23 \mathrm{mJy}$; ours is $92 \mathrm{mJy}$. This comparison is difficult because the shape of $\mathrm{H} \alpha$ is known to be variable, and also the result will depend on seeing, atmospheric transparency, and on the relative amounts 
of nuclear and extended light that are in the extracted spectrum. In spite of these concerns, the comparison does suggest that in May 1996 both the continuum and $\mathrm{H} \alpha$ were stronger by a factor of about four than they were in June 1995. We can also make a comparison at $\mathrm{H} \beta$ by using the results of Osterbrock, Koski, \& Phillips (1976). Osterbrock et al. measure $114 \AA$ for the total equivalent width, $\mathrm{EW}$, of $\mathrm{H} \beta$ and $2.6 \times 10^{-13} \mathrm{erg} \mathrm{cm}^{-2} \mathrm{sec}^{-1}$ for the total flux in broad $\mathrm{H} \beta$. We measure $\mathrm{EW}=89 \AA$ and $\mathrm{F}=27.2 \times 10^{-13} \mathrm{erg} \mathrm{cm}^{-2} \mathrm{sec}^{-1}$. The continuum and broad lines appear to have been exceptionally high in May 1996, about 4 times higher than their values a year earlier and about 10 times higher than their values in 1974 .

Our observed flux at $5530 \AA$ is 26.3 mJy, which gives $\mathrm{V} \approx 12.9$. 3C 382 may even have been the brightest quasar in the sky at that epoch. In NED 3C 382 has flux values ranging from 1.6 to $7.3 \mathrm{mJy}$ at $5530 \AA$, so we saw 3C 382 in April and May 1996 at a historic high. Correcting to rest wavelength and using $0.3 \mathrm{mag}$ for the Galactic extinction gives $\mathrm{M}_{\mathrm{B}} \sim-25.0$. In its brighter phases 3C 382 is a highly-luminous quasar.

\subsubsection{Polarimetry}

In the B-band polarization image (Figure 4) the outer vectors are somewhat circumferential and probably are artifacts due to the strong flux gradient and variable seeing. The polarization values of the central pixels are unreliable due to saturation.

In Figure 2a $p$ rises to the blue, as is common in quasars (Ogle et al. 1999). This is intrinsic and not due to decreasing dilution by galactic starlight, as the starlight fraction is $<10 \%$ (Table 3). The broad lines are polarized substantially less than the continuum, consistent with the hypothesis that we see the continuum source and the BLR more-or-less directly; the former is polarized by local scattering, with possibly a synchrotron contribution, and the latter is weakly polarized by scattering in the BLR itself or farther out. In our work the BLRG $\theta$ 's generally show little correlation with the radio axis (see $\S 10$ ), but 3C 382 is the one closest to parallel, with $\Delta \theta=11^{\circ}$. This might represent a case of scattering in a disk (Antonucci 1984).

The polarization of broad $\mathrm{H} \alpha$ has been studied by Corbett et al. (1998). Although the flux of both the continuum and $\mathrm{H} \alpha$ rose substantially between their measurements and ours, the polarizations are similar. We measure $p=0.85 \%$ and $0.65 \%$ in the continuum at $6500 \AA$ and $7500 \AA$, respectively, and $\sim 0.4 \%$ at the bottom of the dip in $p$ caused by dilution with $\mathrm{H} \alpha$. Corbett et al. (their Figure 1) show $1.05 \%, 0.85 \%$, and $\sim 0.3 \%$. We have $\theta=63^{\circ} \pm 0.4^{\circ}$ with no change at $\mathrm{H} \alpha$, while Corbett et al. have $60.2^{\circ} \pm 1.1^{\circ}$ with perhaps a small change in $\mathrm{H} \alpha$. (Corbett et al. decompose the continuum and $\mathrm{H} \alpha$ but here we give values appropriate to the total flux.) The $\theta$ 's are the same but our values for the continuum $p$ are lower by a small amount. This difference may be significant, but the major result is that the polarization changed little while the flux, both continuum and $\mathrm{H} \alpha$, increased substantially. This suggests that the geometry did not change and shows that there is no appreciable starlight even at the fainter levels, for otherwise the 
polarization would have increased with the decreasing dilution.

Figure 7 shows that the Balmer decrement and spectral index are consistent with little or no dust along the LOS to the continuum source and the BLR. The polarized flux (Figure 1b) has $\alpha=-2.0$, and is substantially steeper than the total flux. $p \times \mathrm{F}_{\lambda}$ is not an achromatic version of $\mathrm{F}_{\lambda}$ but has been bluened.

\section{4. $\quad$ FSC $2217+259$}

The image of FSC $2217+259$ in Figure 4 shows a bright star $6^{\prime \prime} \mathrm{E}$, and there is another $13^{\prime \prime}$ SE. These stars have polarizations $0.4 \% \pm 0.1 \%$ at $\theta=32^{\circ} \pm 1^{\circ}$ and $0.6 \% \pm 0.1 \%$ at $37^{\circ} \pm 1^{\circ}$, respectively. This might well be due to ISP, and so the observations could be corrected for the average, $0.5 \%$ at $34^{\circ}$. The correction is small, $<1^{\circ}$ in $\theta$, and does not materially change either the image or the spectra. We do not correct Figures 1 and 2 for the ISP. Recall that in $\S 4.3$ we noted that $\theta(\lambda)$ is flat for FSC $2217+259$, and that this implies that a substantial foreground polarizing agent is unlikely to exist.

The polarization is high in FSC $2217+259$, rising to nearly $9 \%$ at $4000 \AA$ in $p$, and to $13 \%$ in $p_{\mathrm{c}}$. Figure 2a shows that $\mathrm{H} \alpha$ and $\mathrm{H} \beta$ are polarized more than the neighboring continuum, and $p$ dips in narrow $\mathrm{H} \alpha$. In this respect FSC $2217+259$ is like 3C 234 and other NLRG, but the effect is much weaker and for later discussion we will assume that in FSC 2217+259 the broad Balmer lines are polarized the same as the continuum. The galaxy-subtracted polarization (Figure 3b) is like that of $3 \mathrm{C} 227 ; p_{c}$ is reduced in the narrow [O III] lines and is nearly the same in $\mathrm{H} \alpha$ and the continuum. FSC $2217+259$, however, does not have the $\theta$ rotations in $\mathrm{H} \alpha$ that are displayed by $3 \mathrm{C} 227$.

\subsubsection{Origin of the Polarization}

It is unlikely that the polarization in FSC $2217+259$ is due to extinction by aligned dust grains, for two reasons. First, $p$ has no sign of the Serkowski spectrum, which is gently curved with a peak, usually in V-band (Serkowski, Mathewson, \& Ford 1975). Secondly, the high value of $p, 9 \%$ at $4000 \AA$, is extreme although not unprecedented in stars close to the Galactic plane. VI Cyg 12 , at $\mathrm{b}^{\mathrm{II}}=0.8^{\circ}$, has polarization similar to that of FSC $2217+259$, but is much redder. It is more likely that the polarization in FSC $2217+259$ is due to scattering.

Figure 7a shows that the high Balmer decrement can be explained with 3.8 magnitudes of extinction, but this one-component model leaves us with a very blue intrinsic spectral index, and says nothing about the high polarization. The two-component model in Figure $7 \mathrm{~b}$, with $\mathrm{k}_{\mathrm{s}}=.05$

and $p_{\mathrm{c}}=0.30$ yields $\mathrm{A}_{\mathrm{V}} \sim 3.1 \mathrm{mag}$ and $\alpha_{\mathrm{o}} \sim 1.1$. With these parameters the calculated apparent polarization rises from $4.1 \%$ at $7000 \AA$ to $16.4 \%$ at $4000 \AA$. As with $3 \mathrm{C} 227$, the calculated 
polarization is steeper than the measured curve in Figure $3 \mathrm{~b}$. To match the observations the product $\mathrm{k}_{\mathrm{s}} p_{\mathrm{o}}$ would have to be raised by a factor of about 5 . This seems unacceptably high. The model gives a qualitative sense of the spectrum and polarization but does not match FSC $2217+259$ in detail.

The $\sim 3$ magnitudes of extinction on the direct ray means that if this object were seen along a dust-free direction, it would have an absolute magnitude $\mathrm{M}_{V} \sim-23$. Further, the broad-line ratio $\mathrm{H} \alpha / \mathrm{H} \beta$ drops from 10.2 to 4.3, closer to Case $\mathrm{B}$, upon dereddening. All this is consistent with a quasar seen through dust.

\section{5. $\quad 3 \mathrm{C} 445$}

We do not have a polarization image of 3C 445. The spectropolarimetry was taken at Palomar and the noise level is higher than for the Keck data. 3C 445 is like 3C 382 in having very little starlight; but is different in having strong narrow lines and a redder continuum. The polarization rotation in broad $\mathrm{H} \alpha$ is discussed in $\S 9$. The polarization of the $\mathrm{H} \alpha$ region has also been recently discussed by Corbett et al. (1998). Our values for $\mathrm{F}_{\nu}, p$, and $\theta$ (July 1994) agree well with theirs.

3C 445 has $\mathrm{m}_{\mathrm{B}}=17.0$ (NED) which, with a Galactic extinction correction of 0.2 mag, gives $\mathrm{M}_{\mathrm{B}}=-20.4$. The internal extinction at V-band, from Figure 7, appears to be between about 1.1 and 2.5 mag. Assuming a correction to B-band of $0.3 \mathrm{mag}$, we derive an intrinsic $\mathrm{M}_{\mathrm{B}}$ between -21.8 and $-23.2 \mathrm{mag}$. From this analysis, based on the Balmer decrement and the continuum polarization, we find that 3C 445 could intrinsically be bright enough to fit into the quasar category $\left(\mathrm{M}_{\mathrm{B}}<-23\right)$.

\section{Individual Objects: NLRG}

\section{1. $3 \mathrm{C} 195$}

We first discuss 3C 195, the prototypical NLRG showing broad Balmer lines and spatial symmetry in polarized light.

\subsubsection{Interstellar Polarization}

In Figure 2b $\theta$ rotates about $8^{\circ}$ between 3500 and $7500 \AA$. This might be due to interstellar polarization, as the galactic latitude of $3 \mathrm{C} 195$ is low and the estimated upper limit on ISP is $1.1 \%$ (Table 1). We did not measure stars in the field of 3C 195, but two stars within $3^{\circ}$ have $p \sim 0.6 \%$ at $\theta \sim-35^{\circ}$, and $p \sim 0.9 \%$ at $\theta \sim-13^{\circ}$. (Mathewson \& Ford 1970). Correction for the average,

$p=0.75 \%$ at $\theta=-24^{\circ}$, yields a curve of $\theta$ that tilts a little in the other direction, indicating 
perhaps an over-correction. In addition the [O III] lines become less prominent in $\theta$ than they are in Figure 2, and a $\theta$ rotation in broad $\mathrm{H} \alpha$ becomes noticeable. The partial disappearance of the [O III] lines is a sign that the correction may be useful, but the appearance of broad $\mathrm{H} \alpha$ in $\theta$ seems like an artifact. Altogether, we believe that a correction is needed, one rather smaller than the average shown above. However, given the notorious gradients of ISP in the Galaxy, we do not pursue this farther, since it needs the measurement of stars in proximity to 3C 195. The corrected value of $\theta$ is probably close to $120^{\circ}$. The correction would have negligible effect in $\mathrm{B}$ band because $p$ is high, and Figure 5 is unaffected by it, except in the regions where $p$ is low. The magnitude $p$ (Figure 2) has negligible correction, at all wavelengths except the [O III] lines, because the correction vector (in $q, u$ space) is nearly perpendicular to the measured vector.

\subsubsection{The Polarization Image}

Figure 5c shows the B-Band polarization image. The image shows oppositely directed "fans" of polarization with vectors that are roughly azimuthally directed. This is the sign of a reflection nebula, in which a point source illuminates the surrounding material and the scattered light is polarized perpendicular to the plane of scattering. The fans are roughly co-spatial in projection with the EELR (Cimatti \& di Serego Alighieri 1995). The general interpretation of this pattern is that an anisotropic beam of radiation illuminates and photoionizes the surrounding material in two oppositely directed "cones". The EELR fans are the projected cones of ionization. The beam is scattered by electrons and dust in the EELR and we see a partially polarized version of

the nuclear spectrum. The narrow lines are recombination radiation that is less polarized than the nuclear light because it arises outside (or perhaps partly inside) the scattering region.

Note that the cones are not symmetric. To the NW there appears to be a non-illuminated region that extends in close to the nucleus. To the SE the corresponding region has polarized light, close to the nucleus. This may reflect different $\mathrm{S} / \mathrm{N}$ on the two sides, rather than a real lack of scattered light NW.

In Figure 5c $p$ is weak in the center (3\%), rises rapidly for $2^{\prime \prime}$ to the NE (15\%) and SW (11\%), and then decreases slowly. The strong gradient is somewhat surprising, since a simple model, consisting of optically thin scattering from electrons or dust with density decreasing linearly with radius, gives $p$ that is independent of radius. Figure 5 d shows a contour diagram of the polarized flux, $p \times \mathrm{F}_{\lambda}$, whose axis is oriented at $\mathrm{PA} \approx 35^{\circ}$, rather far from the radio axis at $19.5^{\circ} \cdot p \times \mathrm{F}_{\lambda}$ has two peaks; the stronger one is displaced about $0.5^{\prime \prime} \mathrm{SW}$ of the flux peak, and the other is $2^{\prime \prime} \mathrm{NE}$, where the flux contours are extended and bend $\mathrm{N}$. The reduction in $p$ and $p \times \mathrm{F}_{\lambda}$ at the center is discussed in $\S 5.2$, where we suggest that it is due to the cone geometry, with dilution by starlight also affecting $p$. This reduction in $p$ and $p \times \mathrm{F}_{\lambda}$ is common, as it exists in 4 of the 5 highly polarized NLRG.

A uniformly filled scattering cone will show a polarization at the apex that is perpendicular to 
the axis of the system. In the central $1.3^{\prime \prime}$ of Figure $5 \mathrm{c}$ the vectors have $<\theta>=130.4^{\circ} \pm 3.7^{\circ}$, and we assume they are perpendicular to the axis of the cone, which then is at $\mathrm{PA} \approx 41^{\circ}$. The axis of $p \times \mathrm{F}_{\lambda}\left(35^{\circ}\right)$ is close to this cone axis. A line at $\mathrm{PA}=41^{\circ}$ is within about $2^{\circ}$ of perpendicular to all the vectors along its path. This line is the symmetry axis of the reflection nebula, because of the perpendicular polarization at the apex, and also the close perpendicularity along the line which is not matched elsewhere.

The axis, and the rotation in $\theta$, can be appreciated in Figure 8, which is a color-coded diagram of $\theta$. The rotation is clear in the north, where the colors are roughly radial, and a center can approximately be located where the various sectors come together. This point is the illumination center. It appears to lie between the peaks in $p \times \mathrm{F}_{\lambda}$, and is not at the flux peak, but rather is displaced to the NE by $0.7^{\prime \prime} \pm 0.2^{\prime \prime}$. This situation may be analogous to the displacements in Cygnus A, where the center is hidden by dust (O97). It would be interesting to compare the radio and optical positions for 3C 195 to see if there is an offset, but the optical positional uncertainty is comparable with our measured displacement (di Serego Alighieri et al. 1994).

The pattern in Figure 8 is more regular in the $\mathrm{N}$ than the $\mathrm{S}$, but the axis can be traced across the entire object. In the $\mathrm{E}$ and $\mathrm{W}$ the red regions are roughly perpendicular to the radius, but they do not extend into the central region. It is likely that the nuclear region is complex, as it is

for the Galaxy and for Cygnus A, with broken dust clouds and, perhaps, multiple scattering. It is important also to remember that the polarization images are smoothed by the seeing weighted by the spatial distribution of $p \times \mathrm{F}_{\lambda}$, and so in weakly polarized regions $\theta$ will be distorted towards its value in nearby strong regions.

Note that the value of $\theta$ obtained with slit spectroscopy may depend on the location of the slit and on the extraction window. In general, the vectors are close to perpendicular to the radius, and so, away from the nucleus, the measured $\theta$ will be close to perpendicular to the slit orientation. This means that one must take care in comparing a polarization to a radio axis in an NLRG like 3C 195, because the value of $\theta$ will depend on the details of how it is measured.

\subsubsection{Spectropolarimetry}

For spectropolarimetry we oriented the slit to include the strongly polarized regions shown in Figure 5d. We extracted the spectrum and polarization in the nucleus and in regions to the $\mathrm{NE}$ and SW, but in this paper we only present spectra centered on the nucleus. Subtraction of the galactic light is not enough to flatten $p_{c}$ (Figure 3 ). The corrected polarization rises to the blue and is about $14 \%$ at $3500 \AA$. Also, broad $\mathrm{H} \alpha$ and $\mathrm{H} \beta$ are polarized more strongly than the continuum; this might be due to a second continuum component, FC2, as discussed in $\S 4.2$; see also $§ 8.2$. 


\section{2. $3 \mathrm{C} 33$}

3C 33, a well-studied FR II radio source, is associated with a galaxy having an EELR that extends far outside the galaxy. The velocity field in the EELR has been mapped by Baum, Heckman, \& van Breugel (1990), and an optical synchrotron emission region is coincident with the southern hot spot (Meisenheimer, Yates, \& Röser 1997). The main H $\alpha$ axis is not close to the radio axis; this is a case where the alignment effect is weak. Our B-band image is in Figure 5a. A few emission lines, notably [O II] $\lambda 3727$ and [Ne III] $\lambda 3869$, are in the B-band flux, but the lines amount to no more than $25 \%$ of the total and the image is a good representation of the continuum. The lines themselves are essentially unpolarized and do not contribute to the polarized flux. The B-band contours are elongated in the central few arcseconds, with an axis parallel to that of the $\mathrm{H} \alpha$ image. They become circular outside a diameter of $8^{\prime \prime}$, so it is only close to the nucleus that the continuum displays the elliptical shape, whereas the $\mathrm{H} \alpha$ image is elliptical over its total extent, about $12^{\prime \prime}$.

The polarized regions in Figure 5a are asymmetrical, but close to the nucleus they lie roughly along the continuum and $\mathrm{H} \alpha$ axis. The NE polarization region is much larger than the one to the $\mathrm{W}$. The polarization shows a strong gradient, with a peak value $8.4 \%$ in both the NE and the W. The polarization is low near the nucleus, where $p=0.8 \% \pm 0.1 \%$ within the central arcsecond. This value is low enough that it could be affected by ISP, but the high galactic latitude $\left(-49.3^{\circ}\right)$ suggests that the effect is small. Three stars separated from $3 \mathrm{C} 33$ by $3.5^{\circ}, 5.5^{\circ}$ and $6^{\circ}$ have polarizations $0.2 \%, 0.4 \%$, and $<0.1 \%$, respectively (Mathewson \& Ford 1970). It is likely that the ISP in the direction of 3C 33 is a few tenths of one percent, but we ignore it because (a) in fact we do not discuss the polarization at the nucleus in detail (see $\$ 5.2$ ), and (b) we cannot correct for it without observations of stars close to $3 \mathrm{C} 33$. The weak ISP does not affect the high polarization outside the nuclear region.

The plot of $p \times \mathrm{F}_{\lambda}$ in Figure 5b is closely similar to that of 3C 195 in Figure $5 \mathrm{~d}$. Both objects show separated peaks differing in intensity by approximately $30 \%$, and a low-level extension on one side. The position angle $\theta$ rotates around the nucleus in Figure $5 \mathrm{a}$ and stays roughly perpendicular to the radius. Thus $3 \mathrm{C} 33$ provides another good example of a reflection nebula illuminated by a point source. In the nucleus $\theta=91^{\circ} \pm 3^{\circ}$. This is close to parallel to the $p \times \mathrm{F}_{\lambda}$ axis rather than being perpendicular to it, as in 3C 195. 3C 33 does not contain a clear polarization axis, as 3C 195 does. The radio axis, however, is at $19.5^{\circ}$ which, like the axis in most of the NLRG, is within $20^{\circ}$ of perpendicular to the nuclear $\theta$ (see $\S 10$ ). Draper, Scarrott, \& Tadhunter (1993) report imaging polarimetric observations of $3 \mathrm{C} 33$ in V-band, which contains strong [O III] lines and $\mathrm{H} \beta$. The narrow lines are polarized less than the continuum, however, so the Draper et al. polarization image is dominated by the continuum and can be compared with our B-band image. The results generally are similar, and we confirm the centro-symmetric pattern they report. However, we find a stronger gradient, with low polarization at the nucleus.

Spectropolarimetry of $3 \mathrm{C} 33$ was made with the slit along the major axis of the $\mathrm{H} \alpha$ image 
of Baum et al. (1988; PA $62^{\circ}$ ) as shown in Figure 5b. In Figures 1-3 we show spectra extracted from the region of the NE peak in $p \times \mathrm{F}_{\lambda}, 2^{\prime \prime}$ from the nucleus. This region has $\theta=156^{\circ}$, nearly perpendicular to the slit direction, as expected for a resolved reflection nebula.

3C 33 is an NLRG, but a weak broad line can be seen under $\mathrm{H} \alpha$ in Figure 1a, and broad $\mathrm{H} \alpha$ is the dominant feature in polarized light, Figure 1b. The starlight fraction is high (Table 3) and the corrected flux in Figure 3 shows prominent broad Balmer lines. The polarization corrected for starlight is shown in Figure 3b. In broad $\mathrm{H} \alpha p_{c}$ rises to $\sim 25 \%$; this is the highest value of $p_{c}$ that we have measured. This perhaps is not surprising, as it is for an off-nuclear location where geometric dilution is expected to be smaller than near the nucleus. The continuum polarization is lower, near $12.5 \%$, suggesting that there is a strong FC2 component. The position angle shows no changes at $\mathrm{H} \alpha$ so we can assume that the nuclear continuum and $\mathrm{H} \alpha$ are intrinsically polarized the same. In this case we can follow the analysis of Tran (1995; see eqs 2.5-2.7), and we calculate $\mathrm{FC} 2 / \mathrm{FC} 1 \sim 3$, and the intrinsic polarization of $\mathrm{FC} 1$ and broad $\mathrm{H} \alpha, p_{\mathrm{s}}$, is about $50 \%$. That value is basically calculated at one wavelength, $\mathrm{H} \alpha$, where $p_{c}$ is a maximum. To obtain a feel for the validity of our analysis, we plot in Figure 9 the spectrum of FC2 (the "unpolarized flux spectrum", see Tran 1995, Figure 1) with $p_{\mathrm{s}}$ held constant in wavelength. The top spectrum shows the corrected flux; this is the same as the plot in Fig. 3a. The lower curves show spectra for $p_{\mathrm{s}}=0.3,0.5$, and 0.7. The fit can best be examined in the blue wing of $\mathrm{H} \alpha$, at $6500 \AA$. It is clear that $p_{\mathrm{s}}=0.3$ undercorrects for $\mathrm{FC} 2$, and $p_{\mathrm{s}}=0.7$ overcorrects. A value between 0.4 and 0.6 appears to provide a good average correction over the $\mathrm{H} \alpha$ line. The noise is higher in $\mathrm{H} \beta$, but $p_{\mathrm{s}}=0.5 \pm 0.1$ appears to be valid there also.

Our value for the intrinsic polarization due to scattering, $p_{\mathrm{s}}=50 \% \pm 10 \%$, is higher than values reported earlier. This is at least partly due to the off-nuclear position of the measurement, but 3C 234 appears to have no geometric dilution (Fig 5e), and for it we found $p_{\mathrm{s}}=25 \% \pm 3 \%$ (T95). In a group of 10 Seyfert 2 galaxies Tran (1995) found a maximum value $35 \% \pm 9 \%$. High values of $p_{\mathrm{s}}$, like the one we find in $3 \mathrm{C} 33$, in fact are expected in simple scenarios of scattering and the low values more commonly found have required some explanation (e.g. Miller \& Goodrich 1990).

\section{3. $3 \mathrm{C} 105$}

3C 105 is the only object in our sample that shows no evidence for polarization, with $p<0.8 \%(3 \sigma)$. The galaxy is exceptionally red, with strong stellar absorption lines Tadhunter et al. 1993). The Keck spectra are shown in Figure 10; the bottom panel is the spectrum with a galactic template (corrected for reddening) subtracted. There is no reliable indication of broad lines. 3C 105 has a typical FR II radio morphology, with a weak core and prominent hot spots, and is similar to $3 \mathrm{C} 357$. 


\section{4. $3 \mathrm{C} 135$}

3C 135 has several nearby companions (Figure 6), including one $4^{\prime \prime} \mathrm{SW}$ and a highly-distorted one 14" SW. The HST image (de Koff et al. 1996) also shows a fine-scale extension from the nucleus to the SW. The polarization of 3C 135 is low; even after the strong correction for host galaxian starlight it is only about 3\%, with a large error. Eracleous \& Halpern (1994) did not find a broad component of $\mathrm{H} \alpha$ in 3C 135, and $\mathrm{H} 96$ similarly found narrow but not broad $\mathrm{P} \alpha$. However, although 3C 135 is weakly polarized, broad $\mathrm{H} \alpha$ and (perhaps) $\mathrm{H} \beta$ are visible in $p$ and $p \times \mathrm{F}_{\lambda}$. 3C 321 (Table 4) is similar in not showing broad $\mathrm{P} \alpha$ while showing broad $\mathrm{H} \alpha$ in polarized light.

In the polarization image of 3C 135 (Figure 6) the vectors are stronger than $3 \sigma$ in 10 bins near the nucleus. The value measured near the center is $p=1.5 \% \pm 0.2 \%$ with $\theta=159^{\circ} \pm 3^{\circ}$. The image is taken in B band that contains [O II] $\lambda 3727$ plus starlight in addition to the weak continuum. The spectropolarimetry is noisy in B band but at $5500 \AA$ the value is $p=0.7 \% \pm 0.1 \%$ with $\theta=142.1^{\circ} \pm 4.8^{\circ}$ (Table 3 ). The values are consistent with a polarization that rises to the blue, as is common in NLRG, and they are marginally consistent with $\theta$ being independent of wavelength.

\section{5. $3 \mathrm{C} 234$}

The spectropolarimetry for 3C 234 has been discussed by T95. Figure 5 shows contour diagrams of $\mathrm{F}_{\lambda}$ and $p \times \mathrm{F}_{\lambda} . p$ increases to the East and has a maximum about $1.5^{\prime \prime}$ east of the flux maximum. This gradient is compensated by the fall of $\mathrm{F}_{\lambda}$, so that $p \times \mathrm{F}_{\lambda}$ and $\mathrm{F}_{\lambda}$ have maxima in the same pixel. Figure $5 \mathrm{f}$ shows that the east-west asymmetries in $\mathrm{F}_{\lambda}$ and $p$ combine to produce $p \times \mathrm{F}_{\lambda}$ that is close to symmetric, although it is noticeably thinner north-south than is $\mathrm{F}_{\lambda}$.

The spatial distributions of $p$ and $p \times \mathrm{F}_{\lambda}$ in 3C 234 are different from those in both the BLRG and the other NLRG. The polarization pattern is extended but does not show fans, and at the nucleus $p$ is neither a minimum nor a maximum. The pattern is probably due to scattering of the nuclear continuum, but the geometry seems to be different from that of the other objects. The lack of polarization fans suggests that we are looking inside the cone, but we must be close to the edge because the broad lines are enhanced in polarized light (much more so for 3C 234 than FSC2217+259). It is hard to explain the symmetry in $p \times \mathrm{F}_{\lambda}$ in this case, especially the lack of circumferential vectors to the north and south.

The source 3.5" NE (Figure 5e) was recognized by Cimatti \& di Serego Alighieri (1995) who stated that it is highly polarized at $\mathrm{V}$ band. In our B-band polarization image no bin in that region shows $p>2.5 \sigma$. We integrated over a $1.1^{\prime \prime}$ square on the NE peak and found $p<1.4 \%(3 \sigma)$. Hurt et al. (1999), using HST ultraviolet imaging polarimetry, also fail to detect polarized light from this region, and suggest that it is dominated by an old stellar population, based on the red color. 


\section{6. $3 \mathrm{C} 321$}

The B-band polarization image of $3 \mathrm{C} 321$ is shown in Figure $5 \mathrm{~g}$. The gradient in $p$ is stronger than it is for the other objects; and, indeed, in the central region no individual bin has a measurable polarization stronger than $2.5 \sigma$. The polarization is marginally detected in a $4 \mathrm{x} 4$ bin $\left(0.8^{\prime \prime}\right)$ average in the center: $p=0.8 \% \pm 0.3 \%$. $p$ rises to $9.3 \%$ in the NW and to $7.2 \%$ in the SE.

The vectors are roughly circumferential, indicating scattering of light from a point source. This pattern has already been noted by Draper, Scarrott, \& Tadhunter (1993). In an attempt to find the source of the nuclear light, we analyzed the angle $\psi$ between the local value of $\theta$ and the radius to an assumed center. This procedure has been used before by Wardle, Perley, \& Cohen (1997). When applied to $3 \mathrm{C} 321$, it shows that $\left\langle\left|90^{\circ}-\psi\right|>\right.$ is a minimum at the SE flux peak, where it has the value $6.9^{\circ}$, whereas at the NW peak it is $14.3^{\circ}$. In other words, the vectors point (perpendicularly) more to the SE component than to the NW component, and the source of nuclear light appears to be in the southern component. This is consistent with Filippenko's (1987) result that the compact radio source is in the SE component. 3C 321 appears to be different from Cygnus A, where the center of activity is hidden by dust and lies between the main nuclear components. Close inspection of the polarization vectors shows that deviations are not random, but rather that the vectors are systematically tilted away from the SE nucleus. This can be caused by seeing, combined with the spatial gradients.

Young et al. (1996) found broad $\mathrm{H} \alpha$ in the polarized spectrum of 3C 321 and, by using the Balmer decrement to estimate the reddening, showed that the intrinsic luminosity was sufficient for the nucleus to harbor a hidden quasar. The spectra we show in Figs 1-3 were made with the slit along the optical symmetry axis and with an extraction window $17^{\prime \prime}$ long, that includes essentially all the galaxy seen in Figure 5. Broad $\mathrm{H} \alpha$ is weakly visible in the total flux in Figure 1 , but is prominent in the polarized flux and in the galaxy-corrected flux (Figure 3). Broad $\mathrm{H} \beta$ and $\mathrm{H} \gamma$ also are visible. Figure 5 shows that the polarization varies widely over the galaxy, so the measured values of $p$ and $\theta$ will generally depend on the slit location and the extraction window. The rapid rise of $p$ to the blue in Figure 2 is partly but not completely due to dilution by an old stellar population, as the increase of $p_{\mathrm{c}}$ in $\mathrm{H} \alpha$ shows that FC2 is present also. Tadhunter, Dickson, $\&$ Shaw (1996) attribute this extra continuum component to A stars. The spectrum of polarized flux is steep in 3C 321, with $\alpha=-2.0$ (Figure 1a). This is the steepest of the NLRG, and is as

steep as 3C 382, which also has $\alpha=-2.0$. In the UV the polarization rises to $40 \%$ Hurt et al. 1999).

H96 did not detect broad $\mathrm{P} \alpha$ in $3 \mathrm{C} 321$, but in our observations the broad lines are obvious in $p \times \mathrm{F}_{\lambda}$ (Fig. 1). This shows the importance of complementary observations. We discuss this further in $\S 10.2$. 


\section{7. $3 \mathrm{C} 357$}

The HST image of 3C 357 (de Koff et al. 1996) shows dust lanes and a patch of emission about $2^{\prime \prime} \mathrm{W}$ of the galaxy; we call this region W. On our Keck B-Band image (Figure 6) W is seen as the bulge in the second-brightest contour (20\%). It lies close to the radio axis. The nucleus does not show any polarization, but in $\mathrm{W}$ four vectors survive the $3 \sigma$ cutoff, giving $p \approx 4 \%$. The vectors are closely perpendicular to the radius, suggesting a scattering origin for the polarized light. The spectroscopic slit at $\mathrm{PA}=95^{\circ}$ was centered on the polarized region, which is near the southern edge of region W. Both the nucleus and W show a great deal of starlight (Table 3) and in Figure 3 we show the galaxy-subtracted flux and polarization of $\mathrm{W}$. (The flux is shown on a linear scale because it is so noisy.) The narrow lines of $\mathrm{W}$ are red-shifted with respect to the nucleus, by $607 \mathrm{~km} / \mathrm{sec}$.

Imaging polarimetry of $\mathrm{W}$ gives $4 \%$ in B Band and spectropolarimetry gives $p=1.9 \% \pm 0.3 \%$ at $5500 \AA$. The rise to the blue is due to dilution by starlight, although narrow lines in the B band image should reduce the $\mathrm{B}$ band polarization. The galaxy-corrected polarization $p_{\mathrm{c}} \sim 14 \%$ in the continuum. The noise is low in the strong narrow lines [O III $] \lambda \lambda 4959,5007$ and $(\mathrm{H} \alpha+[\mathrm{N} \mathrm{II}])$ and

$p_{\mathrm{c}}$ drops to near zero in the lines, indicating that they are polarized much less than the continuum, and arise in situ.

From the nuclear spectra (not shown), an upper limit for $p(5500)$ in the nucleus is $0.4 \%(3 \sigma)$. 3C 357 is an unpolarized NLRG like 3C 105 ( $(8.3)$, but contains a fortuitously located scattering region in the form of region $\mathrm{W}$. It may be similar to the off-nuclear scattering region in NGC 1068 (Miller, Goodrich, \& Mathews 1991). There is no evidence of a BLR in 3C 357, although our S/N is poor. It would be worthwhile to repeat these observations, to establish more clearly the presence or absence of broad Balmer lines. This is of importance in understanding the unpolarized NLRG; is the observed lack of broad lines intrinsic, or merely due to a lack of scattering material? The $\mathrm{S} / \mathrm{N}$ of the current data is too low for a definitive statement, but the apparent lack of a scattered BLR is an intriguing suggestion that there may not be a BLR in 3C 357.

\subsection{Cygnus A}

Cygnus A, the prototypical NLRG, is discussed by O97. The fact that it has a reflection nebula and shows broad lines in scattered light gives strong support to the unification by aspect of BLRG and NLRG.

\section{PA Rotations in the Broad Emission Lines}

Figure $2 \mathrm{~b}$ shows intriguing rotations in $\theta$ across broad $\mathrm{H} \alpha$ in $3 \mathrm{C} 227$ and $3 \mathrm{C} 445$; there are smaller rotations in 3C 321 and Cygnus A, and similar effects in other lines in 3C 227. Similar 
rotations have been seen in Seyfert 1 galaxies (Goodrich \& Miller 1994, Martel 1996, Smith et al. 1997) and in broad-absorption-line QSO (Ogle et al. 1999). Evidently, such rotations are common in broad-line objects. In this Section we describe the rotations in 3C 227 and 3C 445.

\section{1. $3 \mathrm{C} 227$}

Figure 11 gives an expanded view of the polarization of 3C 227, showing (a,b) the Stokes parameters $Q_{\text {tot }}=q \times \mathrm{F}_{\lambda}$ and $U_{\text {tot }}=u \times \mathrm{F}_{\lambda}$, and (c) the position angle $\theta$. To study the PA rotations in more detail we decompose the polarized radiation into continuum and line components, with $Q_{\text {tot }}=Q_{\text {cont }}+Q_{\text {line }}$ and $U_{\text {tot }}=U_{\text {cont }}+U_{\text {line }} . Q_{\text {cont }}$ and $U_{\text {cont }}$ are approximated by the smooth curves in Figures 11a and 11b; they are second-order polynomial fits to $Q_{\text {tot }}$ and $U_{\text {tot }}$ in regions free of emission lines. The resulting $\theta_{\text {cont }}$ is the smooth curve in Figure 11c, which is shown together with $\theta_{\text {tot }}=0.5 \arctan \left(U_{\text {tot }} / Q_{\text {tot }}\right)$. In Figure $11 \mathrm{~d}$ the smooth curve is again $\theta_{\text {cont }}$ and the other is $\theta_{\text {line }}=0.5 \arctan \left(U_{\text {line }} / Q_{\text {line }}\right)$. We emphasize that this construction depends critically on the exact curves used for $Q_{\text {cont }}$ and $U_{\text {cont }}$, and a reliable decomposition can only be made with high-S/N data.

In the spectrum of 3C 227 (Fig 1) a weak broad feature is visible at $5700-5900 \AA$. This is a blend of He I $\lambda 5876$, Na I $\lambda \lambda 5890,5896$, Fe VII $\lambda 6087$ and, probably, other lines. (See Thompson 1991 for a discussion of the He and Na lines.) This feature is polarized and is seen in the curves of $Q$ and $\theta$ in Fig. 11. Similarly, the blue wing of $\mathrm{H} \gamma$ contains a contribution from broad He II $\lambda 4686$. We shall refer to these blends simply as He I $\lambda 5876$ and $\mathrm{H} \gamma$.

In Figures 11c and 11d it is clear that broad $\mathrm{H} \alpha, \mathrm{H} \beta, \mathrm{He} \mathrm{I} \lambda 5876$, and possibly $\mathrm{H} \gamma$, have a PA that is different from that of the continuum. $\mathrm{H} \alpha$ and $\mathrm{H} \beta$ are rotated about $20^{\circ}$ from the continuum, and $\theta$ is constant in these lines to within about $10^{\circ} . \mathrm{H} \gamma$ and $\mathrm{He} \mathrm{I}$ are rotated from the continuum in the same direction as $\mathrm{H} \alpha$ and $\mathrm{H} \beta$ and possibly by the same amount, although they are weak and the errors, due mainly to uncertainties in the continuum levels, are large.

The narrow lines are polarized less than the continuum and broad lines, as is usual in these RG. [O III] $\lambda 5007$ appears as a prominent spike in $\theta$, and formally is at $\theta \approx 5^{\circ}$, but we are suspicious of this value, because it occurs in only one pixel. It might be due to noise, or to problems with the spectrum extractions. [S II] appears to show up in $\theta$ and $\theta_{\text {line, }}$ but this may be due to noise as the $[\mathrm{S} \mathrm{II}]$ signal is weak in $Q$ and $U$.

\section{2. $3 \mathrm{C} 445$}

Figure 12 shows the $\mathrm{H} \alpha$ region of $3 \mathrm{C} 445$ in the same construction as for $3 \mathrm{C} 227$, except that

we used a linear fit to the continuum for $Q_{\text {tot }}$. Note that in (c) and (d) the scales differ by a factor of two. The rotation in $\mathrm{H} \alpha$ shows a striking red/blue difference in 3C 445, one lacking in 3C 227. 
The crossover occurs at about $6500 \AA$, which is offset from the narrow-line systemic velocity by $3000 \mathrm{~km} / \mathrm{sec}$. The red side in $\theta_{\text {line }}$ (Figure $12 \mathrm{~d}$ ) is roughly constant at $130^{\circ}$ and is rotated by about $10^{\circ}$ from $\theta_{\text {cont }}$. This component clearly persists to $6820 \AA$ or to the end of the red wing in total flux. Its total velocity width is $15,000 \mathrm{~km} / \mathrm{sec}$. The blue side may persist to $6180 \AA$, although the noise near $6300 \AA$ makes this questionable. Its width is at least $7000 \mathrm{~km} / \mathrm{sec}$ and could be up to $15,000 \mathrm{~km} / \mathrm{sec}$. The value of $\theta_{\text {line }}$ clearly changes across the blue component, from about $190^{\circ}$ to about $170^{\circ}$. The peak difference between the red and blue components is about $70^{\circ}$.

The two values of $\theta_{\text {line }}$ for $\mathrm{H} \alpha$ suggest that we are dealing with two components of the radiation, that are separated in velocity and have different PA's. The blue component (B) is dominant below $6500 \AA$, and the red component $(\mathrm{R})$ is dominant above $6500 \AA$. Inspection of the $p \times \mathrm{F}_{\lambda}$ curve (Figure 1b) shows that $\mathrm{H} \alpha$ has a pronounced break near $6500 \AA$, and so such a decomposition seems natural. $\mathrm{B}$ and $\mathrm{R}$ are separated in velocity by $\sim 5000 \mathrm{~km} / \mathrm{sec}$ and are polarized $\sim 1 \%$ with $\theta$ 's that are $\sim 70^{\circ}$ apart.

\subsection{Interpretation}

The Seyfert 1 galaxies studied by Goodrich \& Miller (1994), Martel (1996) and Smith et al. (1997) show a variety of rotations in the broad lines, and several of their objects show rotations

that are as large and as complicated as those in $3 \mathrm{C} 445$. These authors discuss this phenomenon in terms of two or more components that we see via different scattering regions. The regions must lie fairly close to the BLR, for the PA's to differ substantially. Martel decomposes $\mathrm{F}_{\lambda}$ into gaussians, and the best case is NGC 4151 which yields 7 broad components. He then identifies some of the components with structures in the galaxy (a bar, radio and optical axes) on the basis of PA coincidence. We do not attempt a similar deconstruction for 3C 227 and 3C 445 here, but restrict ourselves to one component of $\mathrm{H} \alpha$ for $3 \mathrm{C} 227$, and two for $3 \mathrm{C} 445$.

In 3C 227 and 3C 445 none of the PA's is close to being either parallel or perpendicular to the radio axis. The polarization does not result from simple scattering on a distribution that is symmetric around the radio axis. What then is the source of the polarization? In 3C 227 we evidently have different lines of sight into the nuclear continuum source and the BLR. These light sources are spatially separated by a few tenths of a parsec, so we can imagine that different scatterers are responsible for the two different polarizations. The LOS is not close to the axis of the dusty torus because the radio source has FR II morphology with a weak core, and the inclination is probably many tens of degrees. Thus, the required asymmetry is plausible. A fraction of the $\mathrm{H} \alpha$ light could be scattered and polarized by dust clouds in or near the BLR, and it is possible that absorption in aligned dust particles in front of (or mixed with) the $\mathrm{H} \alpha$ clouds is responsible for some of the polarization. The dust alignment need not have any simple relation to the polarization of the continuum, which is set much deeper inside the nucleus.

3C 445 is more complex, in that we must account for two components of $\mathrm{H} \alpha$, but that 
also offers more constraints and may limit the range of possible models. There is not enough information to invert the polarimetry data and solve for the location and motion of the $\mathrm{H} \alpha$ clouds. However, we can investigate some simple limiting cases and try to find plausible situations. We assume that the system contains a BLR inside a dusty opaque torus, and that the continuum source is at the center. The LOS skims the edge of the torus and we have a limited view of the interior.

We first note that the motions of the $\mathrm{H} \alpha$ clouds cannot be chaotic, since then the red- and blue-shifted sources would be spatially mixed, and the red and blue components would have the same PA, contrary to observation. Hence the clouds must be in streaming motion. This assumes that the velocity shifts are due to motions of the emitting clouds, and not of the scatterers. Further, the lines cannot be broadened purely by thermal motions in clouds, for in that case the electrons would produce equal red and blue shifts at every velocity.

BLR clouds orbiting inside the torus, near the equatorial plane, provide a scenario which can explain much of the observed asymmetry in the polarization of $\mathrm{H} \alpha$. Consider the case of clouds orbiting in circles in the equatorial plane. The motions are not aimed at us, so the observed high velocities must be due to scattering by clouds that see the full red and blueshifts. In other words, the polarized $\mathrm{H} \alpha$ light that we see has been scattered by material that is close to the orbital plane. This material can form the inner wall of the torus. If the rotating BLR clouds are optically thick they mainly radiate back towards the ionizing source, but still, for circular orbits, half of each emitting hemisphere is visible at the rim and the full range of velocities can be seen in the scattered light. An appropriate combination of inclination angle and shape of the inner wall of the torus can now provide red and blue components that have different PA's. See an example in Figure 13. In this example all clouds move on circles in the same direction. In discussing Mrk 231, Goodrich \& Miller (1994) describe a scenario that is similar to the one we present here. Our scenario only works over a restricted range of inclination angles. If the inclination is low the nuclear region is entirely visible and we see a quasar. At high inclination we see an NLRG with polarization perpendicular to the axis. If the view is near the boundary of the torus the equatorial plane is partially visible (Fig 13) and we see a BLRG with different PA's for the red and blue wings of $\mathrm{H} \alpha$.

This simple model cannot be taken literally, for the situation undoubtedly is more complex. For example, Nishiura, Murayama, \& Taniguchi (1998) have described a scenario, based on X-ray spectroscopy, in which the BLR is in a warped disk that is not coaxial with the accretion disk. However, we believe that our concepts still have some validity. The clouds are in streaming, not chaotic motion. Optically thick clouds in equatorial orbits provide a geometry that meets the observations, although the $3000 \mathrm{~km} \mathrm{sec}^{-1}$ offset to the blue is not readily accounted for. Reverberation studies in Seyfert 1 galaxies (e.g. Koratkar \& Gaskell 1991, Korista et al. 1995) show that circular orbits are preferred over radial orbits, and this provides an extra measure of support for our scenario. 


\section{Discussion}

\subsection{Direct and Scattered Light}

The model in $\S 6$ describes the BLRG radiation as a combination of two components, a reddened direct ray and a polarized and reddened scattered ray. The relative amounts of the two rays fix the spectral index and the amount and spectrum of the polarization. The NLRG have more attenuation of the direct ray, so that the nuclear continuum and broad $\mathrm{H} \alpha$ are not directly seen. They show up in the scattered light, and a weaker component, FC2, also becomes important. The evidence for this is that $p_{\mathrm{c}}$ rises in $\mathrm{H} \alpha$, indicating a diluting unpolarized component in addition to the polarized continuum FC1.

Observationally, the BLRG can be divided into 3 groups that have rather different spectra and polarization. However, these should not be thought of as separate classes of objects, since they are primarily distinguished by differing extinction and inclination angle.

(i) 3C 382 is very blue, has low polarization, and broad $\mathrm{H} \alpha$ is prominent in total flux but not in polarized flux. In its brighter phases, this object is a quasar. The low polarization of $\mathrm{H} \alpha$ can be understood if we have a direct, unattenuated view of the BLR, so that the scattered (polarized) light from the BLR is weak in comparison to the direct ray. Another way of looking at this is to imagine that all BLRG have a more-or-less standard spectrum of $p \times \mathrm{F}_{\lambda}$, as exemplified by $3 \mathrm{C} 227$. 3C 382 additionally has a strong unpolarized component of $\mathrm{H} \alpha$ that dilutes the polarization in the line. This combination leaves the position angle unchanged in the line, as required by Figure $2 \mathrm{~b}$.

(ii) 3C 227 and 3C 445 are highly polarized in the blue. The broad lines are equally prominent in total and polarized flux. PA rotations in $\mathrm{H} \alpha$ show that there are multiple lines of sight to the BLR. We have a view deep into the illuminated cone, but may not directly see the nuclear continuum source or the BLR. The view probably is close to the edge of the torus. These two objects are analogous to the Type 1 Seyferts that have PA rotations, but the RG also have powerful radio jets.

(iii) 3C 109 and FSC 2217+259 are very red, highly polarized, and the polarization of broad $\mathrm{H} \alpha$ is the same as that of the continuum. They are closely similar except for the spectral index of the continuum (Figure 7). FSC $2217+259$ is a quasar seen through about 3 magnitudes of dust extinction (according to $\S 7.4 .1$ ), with a scattered component in which the continuum and broad lines are polarized the same. The net polarization $\left(p_{c}\right)$ is high and rises strongly to the blue; this is due to the reduction in dilution by the heavily-reddened direct component. 3C 109 has been interpreted as being polarized by selective absorption in aligned dust grains, but in view of its similarity to FSC $2217+259$ we think it may also have a scattered component. Observations into the UV would be useful for both these objects.

In any event, 3C 109 and FSC 2217+259 are quasars seen through dust. We are looking deep into the torus, and may have a direct (but attenuated) view of the BLR and the nuclear continuum source. FSC $2217+259$ does not have a compact optical nucleus like the other BLRG, 
and its image of $p \times \mathrm{F}_{\lambda}$ resembles that of $3 \mathrm{C} 234$.

\subsection{Unification}

Our work strongly supports the hidden nucleus hypothesis for NLRG and the unification by aspect of many FR II NLRG, BLRG and quasars, in four ways.

(i) Reflection nebulae, in the form of fans of polarized light, are seen in some NLRG. The polarization vectors are circumferential and accurately define a central hidden continuum source. The fans are roughly co-spatial with the EELR, and the radio axis is usually inside the fans.

(ii) Broad $\mathrm{H} \alpha$ is seen in 6 of the 7 polarized NLRG, showing that they contain a hidden BLR. Our high-quality spectra show this feature directly, but it is much more prominent in polarized light. The seventh polarized object, 3C 357, is the weakest and is unreliable because the atmospheric A band falls on the red wing of $\mathrm{H} \alpha$.

(iii) The intrinsic luminosity of the BLRG in our sample, and also of 4 NLRG, 3C 227 (Prieto et al. 1993), 3C 234 (T95), 3C 321 (Young et al. 1996) and Cyg A (O97), has been investigated in detail. It is sufficient to put all of them in or close to the quasar class.

(iv) In the BLRG the outer reflection nebulae must exist, as they do for the NLRG, but they have not been seen. However, in BLRG the nuclear continuum and broad lines swamp the weaker light from the reflection nebula. A measure of this difference can be gained from Table 3, where the galaxy fractions $f_{g}$ show that the starlight (and presumably the outer scattered light) is weaker in the BLRG than in the NLRG by a factor of 3 to 10 or more. A further reason why the reflection nebulae might be hard to see in the BLRG is foreshortening due to the smaller inclinations. Although the highest-redshift object is 3C 109, a BLRG, the other BLRG have redshifts in the range of the NLRG, and angular size is not a major factor in the visibility of the EELR.

Another commonly-used aspect indicator is $\mathrm{R}$, the ratio of the radio flux density in the core to the lobe flux density. $\log \mathrm{R}$ at $6 \mathrm{~cm}$ wavelength is listed in Table 5 . The mean value of $\log \mathrm{R}$ for the BLRG is -1.1 and for the NLRG it is -2.1 . The difference is highly significant and shows that the BLRG radio axes are closer to the LOS than the NLRG axes, on average.

These results justify the common scenario of an occulting torus that hides the nucleus from direct view but allows collimated nuclear light to be scattered into our LOS. From other aspects the NLRG would look like BLRG and quasars. Our results suggest that most polarized NLRG

contain a hidden BLR. However, we cannot generalize about the fraction of unpolarized objects that might show broad lines because we have only one unpolarized NLRG. We also cannot generalize to find the statistical occurrence of broad lines in all NLRG, for the sample is biased towards objects known in advance to be polarized.

Broad lines should be seen in the IR if the dusty torus picture is correct. H96 looked for 
$\mathrm{P} \alpha$ in a complete sample of $11 \mathrm{FR}$ II radio sources, including 1 quasar, 1 BLRG, and 9 NLRG. Their sample includes 3C 273 which usually is not called FR II because it is not a classical radio double. However, we keep it because it has high luminosity and one visible relativistic jet with an outer lobe. One NLRG, 3C 319, is dominated by starlight and has no apparent H $\alpha$; it was not observed in the IR but we keep it in the sample, which consists of all FR II 3CR objects in the ranges of flux, redshift, latitude, right ascension and declination chosen by H96. We have changed their designation of 3C 234 from BLRG to NLRG. Three of their NLRG, 3C 135, 3C 234, and 3C 321, are in our sample. Table 4 summarizes the combined data sets. Y and N simply indicate whether there is or is not evidence for high polarization, broad $\mathrm{H} \alpha$ and broad $\mathrm{P} \alpha$. We have added the polarization value (N) for 3C 236 from Impey, Lawrence, \& Tapia (1991), because although a galaxy-subtracted value is not available, the measured value is low, $0.2 \pm 0.3 \%$. Table 4 contains 14 NLRG, of which 10 show evidence of broad lines and 4 do not. There is a strong correlation between polarization and the existence of broad lines: 6 of the 7 NLRG with high $p_{c}$ show broad lines, and the 2 with no measurable continuum polarization do not show polarized broad lines. This reinforces the picture seen in our data alone, that polarized objects will show broad lines, either in polarized light or in the IR.

H96 had a complete, if small sample, and the combination of our spectropolarimetric results with their near-IR results is more powerful than either technique alone. This produces a result that is correspondingly more robust. While H96 did not see broad $\mathrm{P} \alpha$ in $3 \mathrm{C} 135$ and 3C 321, we have shown that there is indeed a BLR in these objects. This brings the number of objects known to have a BLR to 8 in the complete H96 sample of 11 FR II objects, or 6 of the 9 NLRG.

Tadhunter et al. (1997) have made a polarization survey of a complete sample of radio galaxies, of which 6 are FR II NLRG. Three of the 6 are polarized, but none shows evidence of broad lines. This seems to contradict our result that polarized NLRG show broad lines, but limits on the strength of the broad lines are not available for their objects. We believe that a sensitive survey of a larger complete sample is warranted.

The question of whether the unpolarized FR II NLRG contain a BLR is important, and unanswered. In particular, the lack of evidence for broad lines in an object should not be construed as showing that it has no BLR. There may be no signs of broad lines because there is no BLR, or because there are no appropriately placed scattering regions. 3C 357 is essentially unpolarized except for a small region (3C 357W) where the HST image shows an off-nuclear emission region. The polarization there is approximately perpendicular to the line to the center (Figure 6), which suggests that it sees a central continuum source. It does not appear to scatter any broad lines, but the low $\mathrm{S} / \mathrm{N}$ does not allow a firm conclusion to be drawn about the existence of a BLR in the nuclear region.

3C 105 is unpolarized but still is a powerful FR II radio galaxy with a strong NLR, and it should have a bright central engine. In this case the lack of continuum polarization may be due to a lack of scattering material. The lack of observed broad lines could be due to the absence of a 
BLR, but alternatively it could also be due to a lack of scattering material. The referee observes that the apparent lack of scattered nuclear light could alternatively be due to obscuration of the scatterers; this is possible but seems unlikely because the obscuring material would have to cover a large area of the ISM. A polarization image of 3C 105 could be used to search for off-nuclear scattering regions which may have fallen outside our slit. R.A. Fosbury points out (private communication) that it also is conceivable that the central black hole is not currently being fed, so that the nuclear continuum source and BLR are weak, but the long time scales associated with the radio source and the NLR keep them going. As far as we are aware, there are no VLBI observations of $3 \mathrm{C} 105$, so there is no structural information on scales smaller than a few hundred pc.

\subsection{The Radio Connection}

In powerful RG the radio axis is assumed to mark the dynamical axis of the central black hole and accretion disk, and it usually is assumed that any obscuring torus has the same axis. In FR II objects the radio jets are nearly straight and the projected axis is well-defined. In Table 5 we give $\mathrm{PA}$ for the radio axis, $\theta$ for the optical polarization, and $\Delta \theta$, the acute angle between them. The value of $\theta$ for $3 \mathrm{C} 33$ is for the nucleus whereas in Table 3 it is for the NE lobe, corresponding to the spectrum in Figure 1; and for 3C 321 it is for the SE flux peak (Figure 5g) whereas in Table 3 it is for the wide spectrum extraction. In the past much attention has been paid to the relation between $\theta$ and the radio $\mathrm{PA}$, and in particular to the relative orientation of the radio axis with the extra-nuclear UV and emission-line light (alignment effect). In 3C 195, 3C 321 and Cygnus A the radio jet is well inside the polarization fans and the torus axis can be taken as coincident with the radio axis, although the torus is far from a smooth circular structure and the illuminated cones must be irregular. In 3C 33 the polarized fans are not coaxial and the collimation mechanism appears to give different illumination patterns on the two sides, even though the radio structure is nearly straight across the nucleus. The inner $6^{\prime \prime}$ of the $p \times \mathrm{F}_{\lambda}$ image (Figure $5 \mathrm{~b}$ ) is nearly

symmetric but the large weak region to the $\mathrm{N}$ is not repeated in the $\mathrm{S}$. In $3 \mathrm{C} 33$ the $p \times \mathrm{F}_{\lambda}$ axis is far $\left(50^{\circ}\right)$ from the radio axis.

The important feature of the polarization image in NLRG is that it shows the reflection nebula. At the nucleus itself the polarization becomes weak but in all cases it is within $24^{\circ}$ of being perpendicular to the radio axis (Table 5). We think this comes about because, when there is sufficient symmetry at the apex, the contributions add up to give an orientation perpendicular to the cone axis, which is close to the radio axis. In 3C 33 the alignment effect is one-sided, but the nuclear polarization is still roughly perpendicular to the radio axis. In this case the polarization rotates strongly and is weak at the nucleus (Figure 5a) so that observations must have high S/N and high angular resolution to be reliable.

Antonucci (1984) noted that the BLRG are mostly polarized with $\theta$ roughly parallel to the radio axis. From Table 5 we see that the BLRG differences do not cluster near $0^{\circ}$ or anywhere 
else. However, there are none near $90^{\circ}$, and the median value of $\Delta \theta$ for the BLRG is $31^{\circ}$, whereas for the NLRG the mean is $77^{\circ}$. See also Hurt et al. (1999) for NLRG measured in the UV; they get essentially the same result. The geometry is simple for the NLRG, because the bright central source is occulted and the scattering takes place far from the nucleus. With the BLRG we have a view deep into the ionization cone, but perhaps not into the nucleus itself. The polarization is predominantly produced by scattering, and if the ISM is irregular a range of $\theta$ is to be expected. The one object that looks like a quasar, $3 \mathrm{C} 382$, has $\Delta \theta=11^{\circ}$, showing that its polarization is close to parallel to the radio axis. In this case we apparently do have a direct view of the nuclear continuum source, and the various models that have been proposed to explain the polarization, typically involving an equatorial disk, may be appropriate.

The FR II radio galaxies have a powerful radio jet that is indicative of magnetic field and energetic particles. Hence we might expect that synchrotron radiation could play a role in the optical emission and polarization. None of our objects shows direct evidence of nuclear synchrotron radiation. However, we believe that they are obscured or mis-directed quasars. Thus if (radio-loud) quasars generally do contain a mis-directed blazar, then our objects should also, and there should be a component of synchrotron emission in the continuum radiation. The direct synchrotron beam is narrow and only seen close to the axis. However, in misdirected objects the beam should illuminate the ISM and there should be a narrow bright scattered component. The only possible sign that we have of this is the polarized off-nuclear emission region in 3C 357, and the fact that it is close to the radio axis lends weight to this interpretation. However, the $\mathrm{S} / \mathrm{N}$ is low in this case, and better observations are needed before this possibility can be discussed.

In their discussion of Cygnus A, O97 noted that the larger and more highly polarized fan is on the west side, and that this is the front side of the galaxy, as determined by radio observations. They commented that this is consistent with increased forward scattering on dust particles. We do not have the conclusive information provided by VLBI for the other NLRG with fans, but still can compare the size and strength of the fans with other indicators. We have 3 potential indicators of sidedness: radio structure, polarization fans, and velocity. Table 6 shows the results. Column 2 gives the side with the larger and stronger polarization fan. As can be seen in Figure 5, these are the regions which have extensions in $p \times \mathrm{F}_{\lambda}$, although the peak values of $p \times \mathrm{F}_{\lambda}$ are on the opposite side. We have individual spectra for the two sides and the blue-shifted side is noted; this side is in front if the material is out-flowing. We also attempted to establish the side in front with a radio image. In Cygnus $\mathrm{A}$ this is unambiguously determined by VLBI. In the others we chose the side that has the more complete and stronger jet, but in $3 \mathrm{C} 33$ we were unable to choose. It appears in Table 6 that the correlations are no more than would be given by chance, and that the Cygnus A result cannot be generalized. 


\section{Summary and Conclusions}

1. We have sensitive Keck spectral and imaging polarimetry on 13 FR II radio galaxies - five BLRG and eight NLRG. One NLRG is unpolarized and the others have continuum polarizations ranging from $0.7 \%$ to $9.6 \%$ at $5500 \AA$ (rest frame). When $p$ is corrected for galactic starlight the polarizations rise to $15 \%$, and in 3C 33 a further correction for $\mathrm{FC} 2$ yields a value $p_{\mathrm{s}} \sim 50 \%$ for the intrinsic polarization of the scattered nuclear light. High values like this are expected from scattering in an optically thin cone.

2. Broad $\mathrm{H} \alpha$ is visible in six of the seven polarized NLRG. In five of them the broad lines can be directly seen in the spectrum of total flux, but they are more prominent in polarized flux. In all the polarized NLRG except 3C 357, and perhaps 3C 135, the polarization of broad $\mathrm{H} \alpha$ after correction for galactic starlight is higher than in the neighboring continuum. This is evidence for an additional polarization-diluting continuum component, called FC2. Its nature is unknown but it could be due to hot stars or brehmsstrahlung.

3. The combination of our work with that of H96 shows that at least $6 / 9$ of a complete, volume-limited sample of FR II NLRG have broad lines, seen either in polarization or $\mathrm{P} \alpha$.

4. $\theta$ (measured at the nucleus) is within $24^{\circ}$ of perpendicular to the radio axis for all the NLRG; the mean value of $|\Delta \theta|=77^{\circ}$. The BLRG are not close to parallel. The median value of $|\Delta \theta|=31^{\circ}$, and only one (3C 382) has $|\Delta \theta|<25^{\circ}$.

5. The B band NLRG polarization images show a symmetric polarization pattern characteristic of a bipolar reflection nebula. These objects contain a BLR and a continuum source which is hidden from us by dust but revealed by the polarization spectra and images. From directions within the scattering cone they would look like BLRG or quasars. Hence, the obscuring torus paradigm unifying Seyfert 1 and 2 galaxies also applies to FR II radio galaxies.

6. A large fraction of polarized NLRG contain a BLR, but we do not know if the correlation between $p$ and the BLR is intrinsic or merely due to the existence of appropriately located scattering material that produces the polarization and also allows the BLR to be recognized. Conversely, we have one unpolarized NLRG that does not show broad lines. We do not know if the BLR is missing or if there is just a lack of scattering material. We are unable to generalize these results to find, for example, the fraction of NLRG that are polarized, as our sample is strongly biased towards objects known in advance to be polarized.

7. There is a hint in the low $\mathrm{S} / \mathrm{N}$ data for 3C 357 that there is no BLR, because the west emission region (W) appears to partly consist of scattered nuclear continuum light, but there is no broad $\mathrm{H} \alpha$. However, this suggestion is not correct if $\mathrm{W}$ is scattering a beamed synchrotron component. There probably is a variation in the luminosity of the BLR in these RG, and that, together with the aspect and extinction, can explain the gradation in broad $\mathrm{H} \alpha$ seen in Figures 1

and 3. We follow Antonucci \& Barvainis (1990) in defining an NLRG as one in which the BLR (and continuum source) is mainly seen by scattering, and a BLRG as one dominated by direct 
nuclear light. By this definition, 3C 234 is an NLRG although broad lines are easily visible in total flux. We suggest that trying to draw careful distinctions here is unimportant, and that it is the geometric and physical properties in the nucleus that are of interest.

8. We propose a model for the BLRG in which the spectra are composed of differing fractions of direct and scattered light, with reddening. The model explains the general blue shape of the polarization spectra, but does not fit 3C 109 and FSC 2217+259 very well. These are the two red BLRG with large extinction on the direct ray.

9. 3C 227 and $3 \mathrm{C} 445$ have marked rotations in $\theta$ in the broad lines. We interpret this to mean that there are multiple lines of sight to the BLR. We have a partial view deep into the nucleus but probably near the edge of the torus. We suggest that the broad-line clouds are in streaming, not chaotic motion, and we describe a simple scenario of clouds in equatorial orbits that can explain much of the observed rotation phenomena in $3 \mathrm{C} 445$.

10. 3C 382 was very bright and was a quasar at our observing epochs. At other epochs it has been several magnitudes weaker and had an easily-visible host galaxy. In this case the names quasar and BLRG have little physical distinction other than to define whether or not the nuclear source is brighter than the stars.

We are grateful to J. Beverly Oke and Judy Cohen, first for building the LRIS and second for the many discussions we had about its use. We are grateful to the instrument specialists at Keck, Tom Bida and Randy Campbell, for their support that often went well beyond the call of duty. We thank I. Browne and M. Marcha for telling us about the high polarization of FSC 2217+259 in advance of publication, and R. Fosbury and J. Vernet for suggestions concerning dust and other matters. We thank R. Antonucci, L. Armus, J. Baker, L. Ferrarese, D. Hines, P. McCarthy and S. di Serego Alighieri for useful discussions. We are grateful to R. Fosbury and S. di Serego Alighieri for assistance with the observations, and thank the referee, Bev Wills, for many useful comments and suggestions. We used the vital NASA Extragalactic Data Base (NED) in this research. The W.M. Keck Observatory is a scientific partnership between the University of California and Caltech, made possible by the generous gift of the W.M. Keck Foundation and support of its president, Howard Keck. This work was partly supported by NSF, Grant AST-9121889, and research by HDT at LLNL was supported by DOE under contract W7405-ENG-48.

\section{REFERENCES}

Antonucci, R.R.J. 1984, ApJ, 278, 499

Antonucci, R. \& Miller, J.S. 1985, ApJ, 297, 21

Antonucci, R.R.J. \& Barvainis, R. 1990, ApJ, 363, L17

Antonucci, R., Hurt, T., \& Kinney, A. 1994, Nature, 371, 313 
Baker, J.C. 1997, MNRAS, 286, 23

Baker, J.C. \& Hunstead, R.W. 1995, ApJ, 452, L95

Barthel, P.D. 1989, ApJ, 336, 606

Baum, S.A., Heckman, T., Bridle, A,. van Breugel, W., \& Miley, G, 1988, ApJS, 68, 643

Baum, S.A., Heckman, T., \& van Breugel, W. 1990, ApJS, 74, 389

Best, P.N., Longair, M.S., \& Röttgering, J.A. 1996, MNRAS, 280, L9

Binette, L., Fosbury, R.A., \& Parker, D. 1993, PASP, 105, 1150

Black et al. 1992, MNRAS, 256, 186

Brindle, C., Hough, J.H., Bailey, J.A., Axon, D.J., Ward, M.J., Sparks, W.B., \& McLean, I.S. 1990, MNRAS, 244, 577

Brotherton, M.S., Wills, B.J., Dey, A., van Breugel, W., \& Antonucci, R. 1998, ApJ, 501, 110

Burns, et al. 1984, ApJ, 283, 515

Burstein, D. \& Heiles, C. 1982, AJ, 87, 1165

Cardelli, J.A., Clayton, G.C., \& Mathis, J.S. 1989, ApJ, 345, 245

Carilli, C.L. 1996, in Cygnus A - Study of a Radio Galaxy, eds C.L. Carilli \& D.E. Harris, (Cambridge Univ Press), p 76

Cimatti, A., di Serego Alighieri, S., Fosbury, R.A.E., Salvati, M., \& Taylor, D. 1993, MNRAS, 264,421

Cimatti, A. \& di Serego Alighieri, S. 1995, MNRAS, 273, L7

Clark, D., Stewart, B.G., Schwartz, H.E., \& Brooks, A. 1983, A\&A, 126, 260

Cohen, M.H., Tran, H.D., Ogle, P.M., \& Goodrich, R.W. 1996, in Extragalactic Radio Sources, eds R. Ekers et al., (Dordrecht: Kluwer), p 223

Cohen, M.H., Vermeulen, R.C., Ogle, P.M., Tran, H.D., \& Goodrich, R.W. 1997, ApJ, 484, 193

Condon, J.J., Anderson, E., \& Broderick, J.J. 1995, AJ, 109, 2318

Corbett, E.A., Robinson, A., Axon, D.J., Young, S., \& Hough, J.H. 1998, MNRAS, 296, 721

de Koff, S. et al. 1996, ApJS, 107, 621

di Serego Alighieri, S., Cimatti, A., \& Fosbury, R.A.E. 1993, ApJ, 404, 584 
di Serego Alighieri, S., Danziger, I.J., Morganti, R., \& Tadhunter, C.N. 1994, MNRAS, 269, 998

di Serego Alighieri, S., Cimatti, A., Fosbury, R.A.E., \& Hes, R. 1997, A\&A, 328, 499

Draper, P.W., Scarrott, S.M., \& Tadhunter, C.N. 1993, MNRAS, 262, 1029

Eracleous, M. \& Halpern, J.P. 1994, ApJS, 90, 1

Fanti et al. 1986, A\&AS, 65, 145

Filippenko, A.V. 1987, in Proc. IAU Symp 124, Observational Cosmology, Eds. A. Hewitt, G. Burbidge, \& G. Fang, p 761

Fosbury, R.A.E., Vernet, J., Villar-Martin, M., Cohen, M., Cimatti, A., di Serego Alighieri, S. 1999, in "ESO Conference on Chemical Evolution from Zero to High Redshift", ESO Astrophysics Symposia, Eds J. Walsh and M. Rosa (Springer), in press

Francis, P.J., Hewett, P.C., Foltz, C.B., Chaffee, F.H., Weymann, R.J., \& Morris, S.L. 1991, ApJ, 373,465

Goodrich, R.W. 1991, PASP, 103, 1314

Goodrich, R.W. 1995, ApJ, 440, 141

Goodrich, R.W. \& Miller, J.S. 1989, ApJ, 346, L21.

Goodrich, R.W. \& Cohen, M.H. 1992, ApJ, 391, 623 (G92)

Goodrich, R.W. \& Miller, J.S. 1994, ApJ, 434, 82

Goodrich, R.W., Cohen, M.H., \& Putney, A. 1995, PASP, 107, 179

Goodrich, R.W., Miller, J.S., Martel, A., Cohen, M.H., Tran, H.D., Ogle, P.M., \& Vermeulen, R.C. 1996, ApJ, 456, L9

Grandi, S.A. \& Osterbrock, D.E. 1978, ApJ, 220, 783

Hardcastle, M.J., Alexander, P., Pooley, G.G., \& Riley, J.M. 1998, MNRAS, 296, 445

Heckmann, T. et al. 1995, ApJ, 452, 549

Heisler, C.A., Lumsden, S.L., \& Bailey, J.A. 1997, Nature, 385,700

Hes, R., Barthel, P.H., \& Fosbury, R.A.E. 1993, Nature, 326, 362

Hill, G.J., Goodrich, R.W., \& DePoy, D.L. 1996, ApJ, 462, 163 (H96)

Hurt, T., Antonucci, R., Cohen, R., Kinney, A., \& Krolik, J. 1999, ApJ, 514, 579

Impey, C.D., Lawrence, C.R., \& Tapia, S. 1991, ApJ, 375, 46 
Impey, C.D., Malkan, M.A., \& Tapia S. 1989, ApJ, 347, 96

Jackson, N. \& Browne, I.W.A. 1990, Nature, 343, 43

Jackson, N. \& Tadhunter, C.N. 1993, A\&A, 272, 105

Kinney, A.L., Bohlin, R.C., Blades, J.C., \& York, D.G. 1991, ApJS, 75, 645

Koratkar, A.P. \& Gatskell, C.M. 1991, ApJS, 75, 719

Korista, K. et al. 1995, ApJS, 97, 285

Kronberg, P.P., Wielebinski, R., \& Graham, D.A. 1986, A\&A, 169, 63

Kwan, J. \& Krolik J.H. 1981, ApJ, 250, 478

Landolt, A.U. 1992, AJ, 104, 340

Laurent-Muehleisen, S.A., Kollgaard, R.I., Ryan, P.J., Feigelson, E.D., Brinkmann, W., \& Siebert, J. 1997, A\&AS, 122, 235

Lawrence, A. 1987, PASP, 99, 309

Leahy, J.P. \& Perley, R.A. 1991, AJ, 102, 537

Leahy, J.P. et al. 1997, MNRAS, 291, 20

Manzini, A., \& di Serego Alighieri, S. 1996, A\&A, 311, 79

Marcha, M.J.M., Browne, I.W.A., Impey, C.D., \& Smith, P.S. 1996, MNRAS, 281, 425

Martel, A.R. 1996, Thesis, University of California, Santa Cruz

Mathewson, D.S. \& Ford, V.L. 1970, MmRAS, 74, 139

McCarthy, P. 1993, ARA\&A, 31, 693

Meisenheimer, K., Yates, M.G., \& Röser, H-J. 1997, A\&A, 325, 57

Miller, J. S. \& Goodrich, R.W. 1990, ApJ, 355, 456

Miller, J. S., Goodrich, R.W., \& Mathews, W.G. 1991, ApJ, 378, 47

Morganti, R., Killeen, N.E.B., \& Tadhunter, C.N. 1993, MNRAS, 263, 1023

Neugebauer, G. et al. 1980, ApJ, 238, 502

Nishiura, S., Murayama, T., \& Taniguchi, Y. 1998, PASJ, 50, 31

Ogle, P.M. et al. 1997, ApJ, 482, L37 (O97) 
Ogle, P.M. 1998, thesis, California Institute of Technology

Ogle, P.M., Cohen, M.H., Miller, J.S., Tran, H.D., Goodrich, R.W., \& Martel, A.R. 1999, ApJS, in press

Oke, J.B. et al. 1995, PASP, 107, 375

Oke, J.B. \& Gunn, J.E. 1982, PASP, 94, 586

Osterbrock, D.E., Koski, A.T., \& Phillips M.M. 1976, ApJ, 206, 898

Osterbrock, D.E. 1989, "Astrophysics of Gaseous Nebulae and Active Galactic Nuclei”, University Science Books, ch. 12

Prieto, M.A., Walsh, J.R., Fosbury, R.A.E., \& di Serego Alighieri, S. 1993, MNRAS, 263, 10

Readhead, A.C.S., Cohen, M.H., Pearson, T.J., \& Wilkinson, P.N. 1978, Nature, 276, 768

Rudy, R.J., Schmidt, G.D., Stockman, H.S., \& Moore, R.L. 1983, ApJ, 271, 59

Schmidt, G.D., Elston, R., \& Lupie, O.L 1992, AJ, 104, 1563

Serkowski, K., Mathewson, S.S., \& Ford, V.L. 1975, ApJ, 196, 261

Simmons, J.F.L. \& Stewart, B.G. 1985, A\&A, 142, 100

Smith, P.S., Schmidt, G.D., Allen, R.G., \& Hines, D.C. 1997, ApJ, 488, 202

Spinrad, H., Djorgovski, S., Marr, J., \& Aguilar, L. 1985, PASP, 97, 932

Storchi-Bergmann, T., Fernandes, R.C. \& Schmitt, H.R. 1998, ApJ, 501, 94

Tadhunter, C.N., Scarrott, S.M., \& Rolph, C. 1990, MNRAS, 246, 163

Tadhunter, C.N., Morganti, R., di Serego Alighieri, S., Fosbury, R.A., \& Danziger, I.J. 1993, MNRAS, 263, 999

Tadhunter, C.N., Dickson, R.D., \& Shaw, M. 1996, MNRAS, 281, 591

Tadhunter, C.N., Dickson, R.D., Morganti, R., \& Villar-Martin, M. 1997, in "Quasar Hosts", ESO/IAC Conference held on Tenerife, September 1996, Eds. D.L. Clements and I. Pérez-Fournon, (Springer) p. 311.

Thompson, K.L. 1991, ApJ, 374, 496.

Tran, H.D. 1995, ApJ, 440, 597

Tran, H.D., Miller, J.S., \& Kay, L.E. 1992, ApJ, 397, 452

Tran, H.D., Cohen, M.H., \& Goodrich, R.W. 1995, AJ, 110, 2597 (T95) 
Tran, H.D., Cohen, M.H., Ogle, P.M., Goodrich, R.W., \& di Serego Alighieri, S. 1998, ApJ, 500, 660

Urry, C.M. \& Padovani, P. 1995, PASP, 107, 803

Veilleux, S., Goodrich, R.W. \& Hill, G.J. 1997, ApJ, 477, 631

Wardle, J.F.C, Perley, R.A., \& Cohen, M.H. 1997, Phys. Rev. Lett., 79, 1801

Wills, B.J., Wills, D., Evans, N.J., Natta, A., Thompson, K.L., Breger, M., \& Sitko, M.L. 1992, ApJ, 400, 96

Young, S. et al., 1996, MNRAS, 279, L72

Zirbel, E.L. \& Baum, S.A. 1995, ApJ, 448, 521 
Fig. 1.- (a) (left panels): Total flux spectra of 11 FR II radio galaxies. The top 5 objects are BLRG, the bottom 6 are NLRG. Spectra are arranged in order of decreasing visibility of broad $\mathrm{H} \alpha$. Note the reddening sequence of the BLRG. The NLRG continua are dominated by starlight from the host galaxies. The logarithmic ordinate has units $10^{-15} \mathrm{erg} \mathrm{sec}^{-1} \mathrm{~cm}^{-2} \AA^{-1}$. (b) (right panels): Polarized flux $\equiv p \times \mathrm{F}_{\lambda}$. Units are $10^{-17} \mathrm{erg} \mathrm{sec}^{-1} \mathrm{~cm}^{-2} \AA^{-1}$. Note that polarized broad lines appear in both BLRG and NLRG. The gaps in 3C 445 and 3C 109 spectra are caused by the dichroic reflector at the Hale Telescope.

Fig. 2.- (a) (left panels): Fractional polarization $p$ of the radio galaxies. In most cases, $p$ increases to the blue and falls in the narrow emission lines. Increased polarization across broad $\mathrm{H} \alpha$ is an indication of diluting continuum sources. (b) (right panels): Position angle $\theta$ of the polarization. $\theta$ is fairly constant with wavelength for most objects. However, note the rotations across broad $\mathrm{H} \alpha$ in 3C 227 and $3 \mathrm{C} 445$.

Fig. 3. - Galaxy-corrected spectra. See Table 3 for the galaxy fraction at $5500 \AA$. (a) (left panels): Flux $\mathrm{F}_{\lambda, c} \mathrm{~F}_{\lambda, c}$ (plotted logarithmically except for $3 \mathrm{C} 357 \mathrm{~W}$; see text). Note that broad $\mathrm{H} \alpha$ is more easily visible after galaxy subtraction. (b) (right panels): galaxy-corrected polarization $p_{\mathrm{c}}$. Polarization rises to the blue even after galaxy correction. The polarization increases at broad $\mathrm{H} \alpha$, an effect attributed to FC2 (see text).

Fig. 4.- BLRG polarization images, B band. Note that the polarization is concentrated in an unresolved nucleus. All images have north up and east to the left. Total flux contours for 3C 382, 3C 109 and 3C 227 are 0.2, 0.4, 1, 5, 50\% of peak; and for FSC 2217+259 they are 5, 10, 20, 50\% of peak. These images are binned $2 \mathrm{x} 2$ from the original data and the scale is $0.43^{\prime \prime}$ per bin, or $2^{\prime \prime}$ per tick. A $1^{\prime \prime}$ polarization vector represents 5, 17, 10, and $20 \%$ polarization for 3C 382, 3C 109, 3C 227, and 2217+259, respectively. The vectors are suppressed for $P<3 \sigma$ (3.5 for 3C 382). The central pixel of $3 \mathrm{C} 382$ is saturated. The axis of the radio structure is marked $\mathrm{R}$.

Fig. 5.- NLRG polarization images, B band. In contrast to the BLRG, the polarized emission is extended and shows a fan structure in some cases. Top: contour diagrams of $\mathrm{F}_{\lambda}$ and the polarization vectors, with radio axis marked $\mathrm{R}$. Bottom: contour diagrams of $p \times \mathrm{F}_{\lambda}$ with slit marked S. Scale as in Figure 4. Contours, in percent of the peak, are a $(1.5,3,8,20,50)$, $\mathrm{b}(5,15,30,50,80), \mathrm{c}(1,2,5,15,50), \mathrm{d}(10,20,40,65,85), \mathrm{e}(0.5,1,2,10,50), \mathrm{f}(1,2,5,15,50), \mathrm{g}(1,2,4,10,20,50)$, $\mathrm{h}(10,25,40,70,90)$. A $1^{\prime \prime}$ polarization vector represents $17,25,33$, and $20 \%$ polarization for a, c, e, and $\mathrm{g}$, respectively.

Fig. 6. - NLRG polarization images, B band. Scale as in Figure 4. Total flux contours, in percent of the peak, are $3 \mathrm{C} 135(1,2,5,15,50), 3 \mathrm{C} 357(3,5,10,20,50)$. A $1^{\prime \prime}$ polarization vector represents $10 \%$ and $8 \%$ polarization for $3 \mathrm{C} 135$ and $3 \mathrm{C} 357$, respectively.

Fig. 7.- (a) Broad-line Balmer decrement vs galaxy-corrected continuum flux ratio for the singlescreen model in $\S 6.1$. Four reddening lines for recombination Case B are shown (solid), with $\alpha_{\mathrm{o}}=-0.5,0.0,0.5$, and1.0. Lines of constant extinction are shown dotted; the extinctions run from 
$\mathrm{A}_{\mathrm{V}}=0$ to 3.5 mag. (b) Galaxy-corrected polarization at $5500 \AA$ (rest) vs flux ratio for the twocomponent model in $\S 6.2$. Solid and dotted lines represent the same quantities as in (a). (c) Balmer decrement vs flux ratio for the two-component model. Solid and dotted lines represent the same quantities as in (a).

Fig. 8. - 3C 195 polarization position angle $\theta$. Note the line of constant position angle at $\theta \simeq 41^{\circ}$ (yellow), which marks the symmetry axis of the reflection nebula. The $\theta$ rotation is apparent in the north, but less so in the south. The data are suppressed for $p<3 \sigma$. Colors are coded as follows: indigo $=84^{\circ}$, blue $=92^{\circ}$, green $=111^{\circ}$, light green $=119^{\circ}$, yellow $=137^{\circ}$, and deep red $=155^{\circ}$.

Fig. 9.- Top spectrum: 3C 33 corrected for Galactic reddening and for a population of old stars. Bottom spectra show the results of correcting for FC2 with $p_{\mathrm{s}}=0.3,0.5$ and 0.7 (see text). The bottom 3 spectra are binned to $20 \AA$.

Fig. 10.- Top: original, uncorrected spectrum of 3C 105. Bottom: dereddened, galaxy-subtracted spectrum of $3 \mathrm{C} 105$.

Fig. 11. - 3C 227 separated into continuum and emission line components. (a) $Q_{\text {tot }}$ and $Q_{\text {cont }}$. (b) $U_{\text {tot }}$ and $U_{\text {cont }}$. (c) $\theta=0.5 \arctan \left(U_{\text {tot }} / Q_{\text {tot }}\right)$ and $\theta_{\text {cont }}=0.5 \arctan \left(U_{\text {cont }} / Q_{\text {cont }}\right)$. Note the strong rotations across the broad lines. (d) $\theta_{\text {line }}$ and $\theta_{\text {cont }}$ (see text). Tick marks indicate $\mathrm{H} \gamma, \mathrm{H} \beta$, [O III] $\lambda \lambda 4959,5007, \mathrm{HeI} \lambda 5876, \mathrm{H} \alpha$, and [S II] $\lambda \lambda 6716,6731$.

Fig. 12.- 3C 445 separated into continuum and emission line components, as in Figure 11. Tick marks indicate $\mathrm{H} \alpha$ and $[\mathrm{S} \mathrm{II}] \lambda \lambda 6716,6731$.

Fig. 13. - Schematic diagram showing the partially-visible equatorial plane of 3C 445. Broad H $\alpha$ clouds orbit on a circle inside an elliptical torus. Red and blue shifted components ( $\mathrm{R}$ and $\mathrm{B}$ ) are scattered and seen with a PA difference of about $30^{\circ}$. When integrated over the visible sector of the torus, red and blue components have the same intensity and fractional polarization, with different position angles. 
Table 1. Low Redshift Radio Galaxies

\begin{tabular}{clccccc}
\hline \hline IAU & \multicolumn{1}{c}{ Name } & Type $^{\mathrm{a}}$ & $\mathrm{z}^{\mathrm{b}}$ & $\mathrm{b}^{\mathrm{II}}(\mathrm{deg})$ & ISP $_{\max }(\%)$ & \multicolumn{1}{c}{ Refs } \\
\hline $0106+130$ & 3C 33 & $\mathrm{~N}$ & 0.0592 & -49.3 & $<0.3$ & $1,2,18$ \\
$0404+035$ & 3C 105 & $\mathrm{~N}$ & 0.0890 & -33.6 & 1.3 & \\
$0410+110$ & 3C 109 & $\mathrm{~B}$ & 0.3056 & -27.8 & 2.3 & $3,4,5,6$ \\
$0511+008$ & 3C 135 & $\mathrm{~N}$ & 0.1274 & -21.0 & 1.1 & \\
$0806-103$ & 3C 195 & $\mathrm{~N}$ & 0.1100 & 12.0 & 1.1 & 7,8 \\
$0945+076$ & 3C 227 & $\mathrm{~B}$ & 0.0862 & 42.3 & $<0.3$ & $1,3,8,9$ \\
$0958+290$ & 3C 234 & $\mathrm{~N}$ & 0.1848 & 52.7 & $<0.3$ & $1,3,4,6,7,10,18$ \\
$1529+242$ & 3C 321 & $\mathrm{~N}$ & 0.0961 & 53.9 & 0.4 & $2,4,7,11,18$ \\
$1726+318$ & 3C 357 & $\mathrm{~N}$ & 0.1661 & 30.6 & 0.5 & \\
$1833+326$ & 3C 382 & $\mathrm{~B}$ & 0.0579 & 17.4 & 0.7 & $1,3,9$ \\
$1957+406$ & Cyg A & $\mathrm{N}$ & 0.0561 & 5.8 & $\ldots$ & $12,13,14,15,16,18$ \\
$2217+259$ & FSC $2217+259$ & $\mathrm{~B}$ & 0.0850 & -25.4 & 0.8 & 17 \\
$2221-023$ & 3C 445 & $\mathrm{~B}$ & 0.0562 & -46.7 & 0.4 & $1,3,6,9$ \\
\hline
\end{tabular}

${ }^{\mathrm{a}} \mathrm{N}=\mathrm{NLRG}, \mathrm{B}=\mathrm{BLRG}$

${ }^{b}$ Redshifts from NED

Note. - References.-(1) Antonucci 1984; (2) Draper et al 1993; (3) Rudy et al 1983; (4) Cimatti et al 1993; (5) Goodrich \& Cohen 1992; (6) Brindle et al 1990; (7) Cimatti \& di Serego Alighieri 1995; (8) di Serego Alighieri et al 1997; (9) Corbett et al 1998; (10) Tran et al 1995; (11) Young et al 1996; (12) Goodrich \& Miller 1989; (13) Tadhunter et al 1990; (14) Jackson \& Tadhunter 1993; (15) Antonucci et al 1994; (16) Ogle et al 1997; (17) Marcha et al 1996; (18) Hurt et al 1999. 
Table 2. Observations

\begin{tabular}{lccccc}
\hline \hline Object & \multicolumn{2}{c}{$\begin{array}{c}\text { SPECTROSCOPY } \\
\text { Exp(sec) }\end{array}$} & Slit PA & Date & Exp(sec) \\
& Date & & & & \\
\hline 3C 33 & & & & & \\
3C 105 & 1096 & 1500 & 62 & 1096 & 1200 \\
3C 109 & 1295 & 2880 & $29^{\mathrm{a}}$ & $\ldots$ & $\ldots$ \\
3C 135 & $1190^{\mathrm{p}}, 191^{\mathrm{p}}$ & 8400 & $90^{\mathrm{b}}$ & 1295 & 2400 \\
3C 195 & 1094,1295 & 3600,5280 & 165,0 & 1096 & 2400 \\
3C 227 & 1295 & 6720 & $35^{\mathrm{a}}$ & 1295,496 & 2400,2400 \\
3C 234 & 1294 & 1920 & 120 & 1295 & 1440 \\
3C 321 & 1094 & 4800 & 90 & $1295^{\mathrm{m}}, 496$ & 2880,2400 \\
3C 357 & 596 & 3600 & 130 & 496 & 1200 \\
3C 382 & 596 & 3600 & 95 & 496 & 2400 \\
Cyg A & 596 & 1920 & 90 & 496 & $120^{\mathrm{s}}$ \\
FSC 2217+259 & 794,1096 & 3600,7920 & 35,101 & 1096 & 3600 \\
3C 445 & 795 & 1200 & 140 & 1295 & 240 \\
\hline
\end{tabular}

${ }^{\mathrm{a}}$ slit width $1.5^{\prime \prime}$

${ }^{\mathrm{b}}$ slit width $2^{\prime \prime}$

${ }^{\mathrm{m}}$ possibly contaminated by moonlight

pPalomar

${ }^{\mathrm{s}}$ saturated 
Table 3. Polarization

\begin{tabular}{lcccccc}
\hline \hline \multicolumn{1}{c}{ Object } & Bin $^{\mathrm{a}}$ & Win $\left({ }^{\prime \prime}\right)^{\mathrm{b}}$ & $p^{\mathrm{c}}(\%) \pm$ & $\theta^{\mathrm{d}} \pm$ & $f_{g}{ }^{\mathrm{e}}$ & $p_{\mathrm{c}}{ }^{\mathrm{f}}(\%)$ \\
\hline 3C 33NE & 8 & 2.4 & 3.10 .4 & 150.52 .2 & 0.74 & 11.9 \\
3C 105 & $\ldots$ & 8.6 & 0.00 .3 & $\ldots$ & 0.78 & $<2.7$ \\
3C 109 & 4 & 5 & 6.80 .2 & 170.50 .6 & $\ldots$ & $\ldots$ \\
3C 135 & 8 & 2.2 & 0.70 .1 & 142.14 .8 & 0.85 & 4.7 \\
3C 195 & 4 & 1.9 & 2.30 .1 & 128.10 .9 & 0.62 & 6.1 \\
3C 227 & 4 & 3.7 & 2.30 .1 & 41.60 .7 & 0.30 & 3.3 \\
3C 234 & 4 & 2.2 & 9.60 .2 & 156.30 .4 & 0.35 & 14.8 \\
3C 321 & 6 & 17.2 & 1.10 .1 & 40.42 .7 & 0.66 & 3.2 \\
3C 357w & 8 & 2.2 & 1.90 .3 & 24.06 .6 & 0.87 & 14.6 \\
3C 382 & 4 & 5.4 & 1.00 .0 & 63.00 .4 & $<0.10$ & 1.0 \\
Cyg A & 4 & 7.7 & 2.30 .1 & 8.51 .7 & 0.70 & 7.7 \\
FSC 2217+259 & 4 & 4.3 & 7.00 .2 & 43.00 .5 & 0.28 & 9.7 \\
3C 445 & 4 & 8 & 2.10 .1 & 142.01 .0 & $<0.10$ & 2.1 \\
\hline
\end{tabular}

${ }^{a}$ number of bins averaged together for Fig 2 and for the polarized flux in Fig 3

${ }^{b}$ Width of extraction window for spectra

${ }^{\mathrm{c}}$ polarization percentage averaged over $5400-5600 \AA$ (rest frame)

${ }^{\mathrm{d}}$ position angle of electric vector, averaged over 5400 - $5600 \AA$ (rest frame)

etemplate galaxy fraction at $5500 \AA$

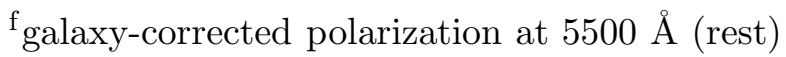


Table 4. Broad line visibility

\begin{tabular}{|c|c|c|c|}
\hline & $\begin{array}{c}\text { High } \\
p_{\mathrm{c}}{ }^{\mathrm{a}}\end{array}$ & $\begin{array}{c}\text { Broad } \\
\mathrm{H} \alpha^{\mathrm{b}}\end{array}$ & $\begin{array}{l}\text { Broad } \\
\qquad \alpha^{\mathrm{c}}\end{array}$ \\
\hline \multicolumn{4}{|l|}{ QUASAR } \\
\hline $3 \mathrm{C} 273$ & $\mathrm{~N}^{\mathrm{d}}$ & $\mathrm{Y}$ & $\mathrm{Y}$ \\
\hline \multicolumn{4}{|l|}{ BLRG } \\
\hline $3 \mathrm{C} 109$ & $\mathrm{Y}$ & $\mathrm{Y}$ & $\ldots$ \\
\hline $3 \mathrm{C} 227$ & $\mathrm{Y}$ & $\mathrm{Y}$ & $\ldots$ \\
\hline 3C 303 & $\ldots$ & $\mathrm{Y}$ & $\mathrm{Y}$ \\
\hline 3 C 382 & $\mathrm{~N}$ & $\mathrm{Y}$ & $\ldots$ \\
\hline FSC $2217+259$ & $\mathrm{Y}$ & $\mathrm{Y}$ & $\ldots$ \\
\hline $3 \mathrm{C} 445$ & $\mathrm{~N}$ & $\mathrm{Y}$ & $\ldots$ \\
\hline \multicolumn{4}{|l|}{ NLRG } \\
\hline 3 C 33 & $\mathrm{Y}$ & $\mathrm{Y}$ & $\ldots$ \\
\hline 3C 105 & $\mathrm{~N}$ & $\mathrm{~N}$ & $\ldots$ \\
\hline 3C 135 & $\mathrm{Y}$ & $\mathrm{Y}$ & $\mathrm{N}$ \\
\hline 3C 184.1 & $\cdots$ & $\mathrm{N}^{\mathrm{e}}$ & $\mathrm{Y}$ \\
\hline 3C 195 & $\mathrm{Y}$ & $\mathrm{Y}$ & $\ldots$ \\
\hline 3C 219 & $\ldots$ & $\mathrm{Y}^{\mathrm{e}}$ & $\mathrm{Y}$ \\
\hline $3 \mathrm{C} 223$ & $\ldots$ & $\mathrm{Y}^{\mathrm{e}}$ & $\mathrm{Y}$ \\
\hline 3C 234 & $\mathrm{Y}$ & $\mathrm{Y}$ & $\mathrm{Y}$ \\
\hline 3C 236 & $(\mathrm{~N})$ & $\ldots$ & $\mathrm{N}$ \\
\hline 3C 321 & $\mathrm{Y}$ & $\mathrm{Y}$ & $\mathrm{N}$ \\
\hline 3C 319 & $\ldots$ & $\mathrm{N}$ & $\cdots$ \\
\hline 3C 327 & $\ldots$ & $\ldots$ & $\mathrm{N}$ \\
\hline 3C 357 & $\mathrm{Y}$ & $\mathrm{N}$ & $\ldots$ \\
\hline Cyg A & $\mathrm{Y}$ & $\mathrm{Y}$ & $\ldots$ \\
\hline
\end{tabular}

${ }^{\mathrm{a}} p_{\mathrm{c}} \geq 3 \%$

${ }^{\mathrm{b}} \mathrm{Broad} \mathrm{H} \alpha$ detected in total or polarized flux

${ }^{\mathrm{c}}$ Broad $\mathrm{P} \alpha$ detected in total flux (Hill, Goodrich, \& DePoy 1996)

dImpey, Malkan, \& Tapia 1989

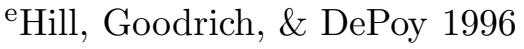


Table 5. Radio and Optical Data

\begin{tabular}{|c|c|c|c|c|c|}
\hline Name & $\log \mathrm{R}^{\mathrm{a}}$ & $\begin{array}{c}\text { Radio Axis } \\
\qquad \mathrm{PA} \pm\end{array}$ & ref & $\begin{array}{c}\text { Optical Pol } \\
\theta \pm\end{array}$ & $\begin{array}{c}\Delta \theta \\
(\mathrm{deg})\end{array}$ \\
\hline \multicolumn{6}{|l|}{ BLRG } \\
\hline $3 \mathrm{C} 109$ & -0.58 & 144.06 & 1 & 170.50 .6 & 26 \\
\hline $3 \mathrm{C} 227$ & -1.90 & 84.57 & 2 & 41.60 .7 & 43 \\
\hline 3C 382 & -1.10 & $52.0^{\mathrm{b}}$ & 3 & 63.00 .4 & 11 \\
\hline FSC $2217+259$ & -0.40 & 158.00 .5 & 4 & 43.00 .5 & 65 \\
\hline $3 \mathrm{C} 445$ & -1.41 & 173.03 .5 & 5 & 142.01 .0 & 31 \\
\hline \multicolumn{6}{|l|}{ NLRG } \\
\hline $3 \mathrm{C} 33$ & -2.25 & 19.51 & 6 & $91.0^{\mathrm{c}} 3.1$ & 72 \\
\hline 3C 105 & -2.24 & 125.5 & 5 & $\ldots$ & $\ldots$ \\
\hline 3C 135 & $-2.72^{\mathrm{d}}$ & 76.03 & 5 & 142.14 .8 & 66 \\
\hline 3C 195 & -1.46 & 19.52 .5 & 2 & 128.11 .4 & 71 \\
\hline $3 \mathrm{C} 234$ & -1.16 & 67.05 & 7 & 156.30 .4 & 89 \\
\hline 3C 321 & -1.78 & 134.50 .7 & 1 & $61.0^{\mathrm{e}} 2.0$ & 74 \\
\hline 3C 357 & -2.21 & $110.0^{\mathrm{f}}$ & 8 & 24.06 .6 & 86 \\
\hline Cyg A & -3.37 & 284.02 & 9 & 8.51 .0 & 84 \\
\hline
\end{tabular}

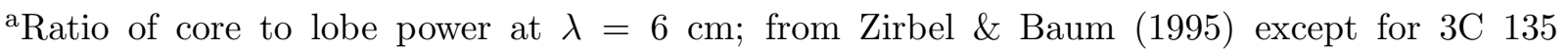
(Hardcastle et al. 1998) and FSC 2217+259 (Laurent-Muehleisen et al. 1997).

${ }^{\mathrm{b}} \mathrm{PA}$ from jet in NE lobe

${ }^{\mathrm{c}} \theta$ at the nucleus

${ }^{\mathrm{d}} \lambda=3.6 \mathrm{~cm}$

${ }^{\mathrm{e}} \theta$ at the SE flux peak

${ }^{\mathrm{f}} \mathrm{PA}$ from SE lobe

Note. - References: (1) Baum et al 1988; (2) Morganti et al 1993; (3) Black et al 1992; (4) Condon et al 1995; (5) Leahy et al 1997; (6) Leahy and Pearly 1991; (7) Burns et al 1984; (8) Fanti et al 1986; (9) Carilli et al 1996. 
Table 6. Scattering Cone, Outflow, and Radio Jet Sidedness

\begin{tabular}{lccc}
\hline \hline & Pol Fan $^{\mathrm{a}}$ & Blue Shift $^{\mathrm{b}}$ & Radio Jet $^{\mathrm{c}}$ \\
\hline 3 C 33 & $\mathrm{NE}$ & $\mathrm{NE}$ & $\ldots$ \\
$3 \mathrm{C} 195$ & $\mathrm{NE}$ & $\mathrm{SW}$ & $\mathrm{SW}$ \\
3 C 321 & $\mathrm{NW}$ & $\mathrm{SE}$ & $\mathrm{NW}$ \\
Cyg A & $\mathrm{W}$ & $\mathrm{W}$ & $\mathrm{W}$ \\
\hline
\end{tabular}

aside with larger polarized fan

${ }^{\mathrm{b}}$ Side with blue-shifted narrow emission lines

${ }^{\mathrm{c}}$ Side with stronger jet, References in Table 5 
TOTAL FLUX

POLARIZED FLUX

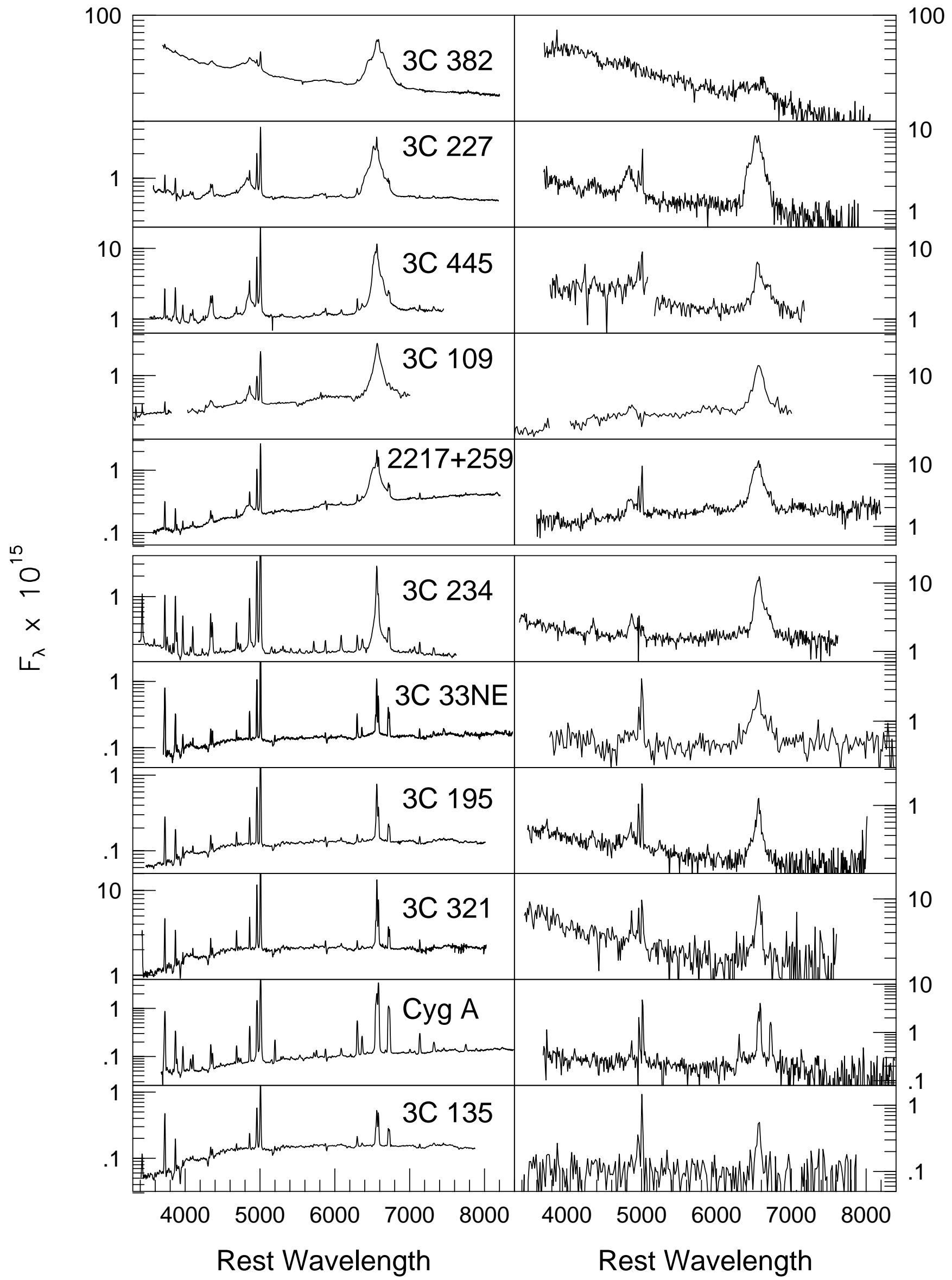


POLARIZATION

POSITION ANGLE

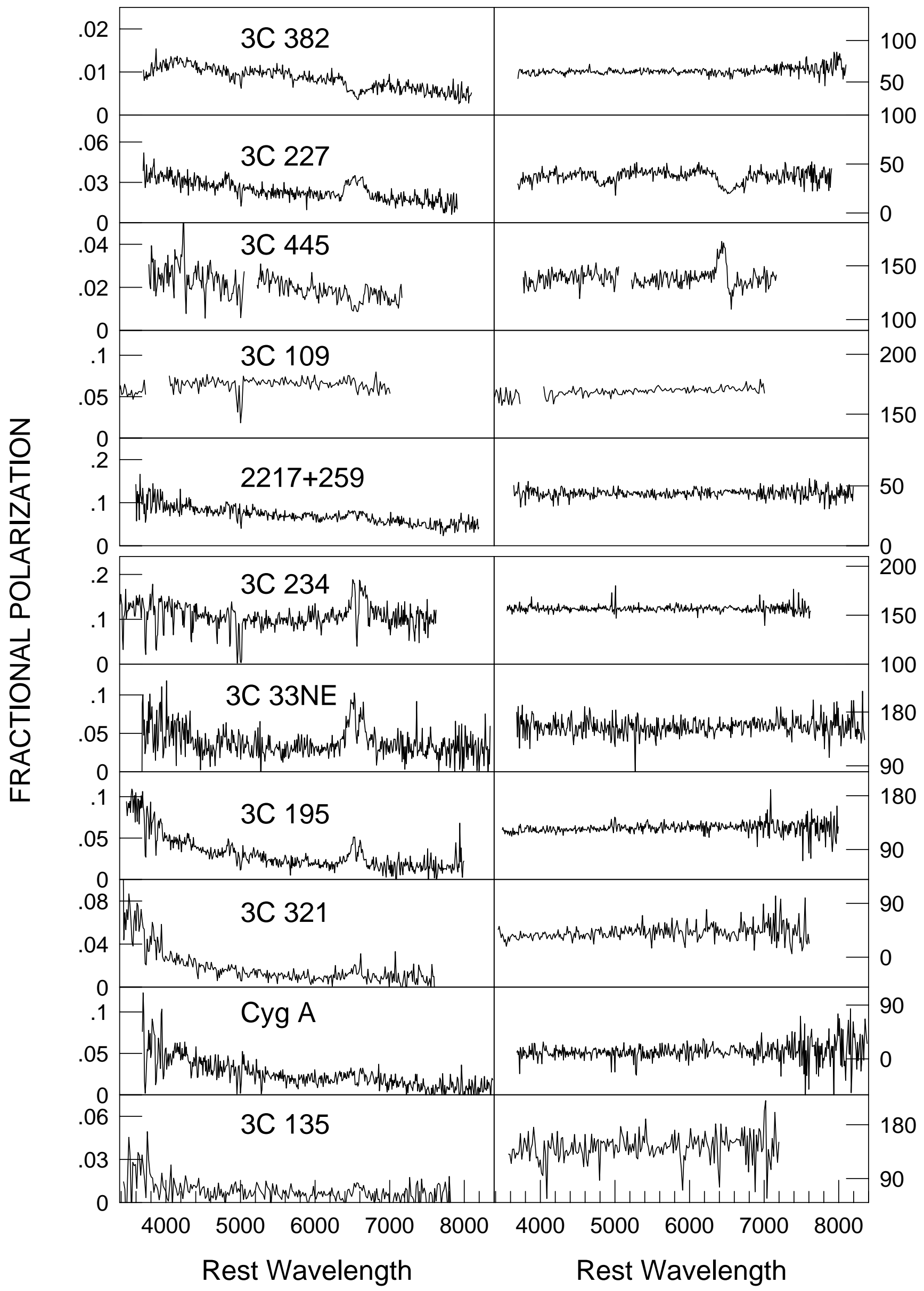

क
0
0
0
0
0
$\Psi$
$\overline{0}$
$z$
$z$
0
$\frac{1}{5}$
0
0 
Corrected $F_{\lambda}$

Corrected Polarization

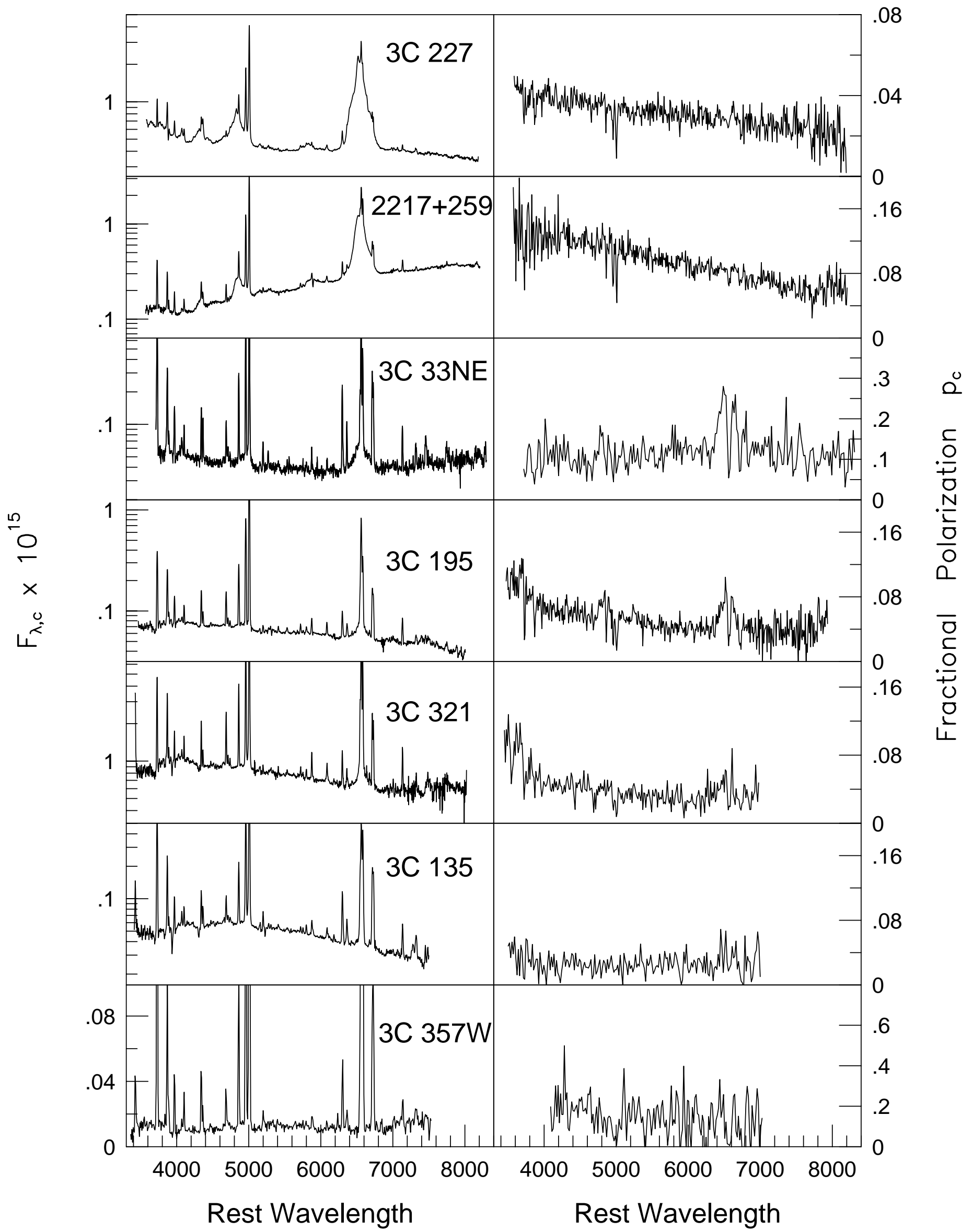




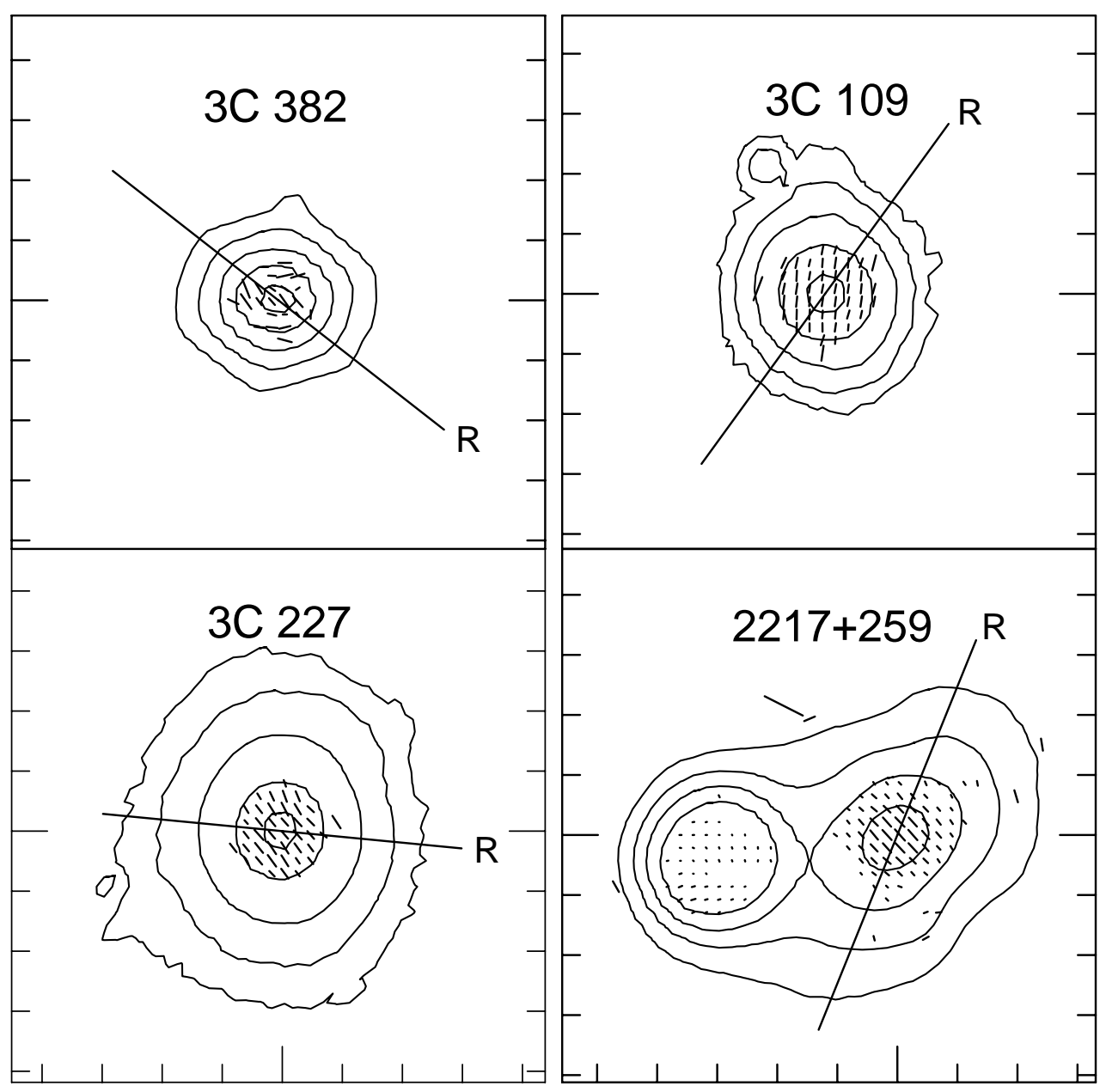



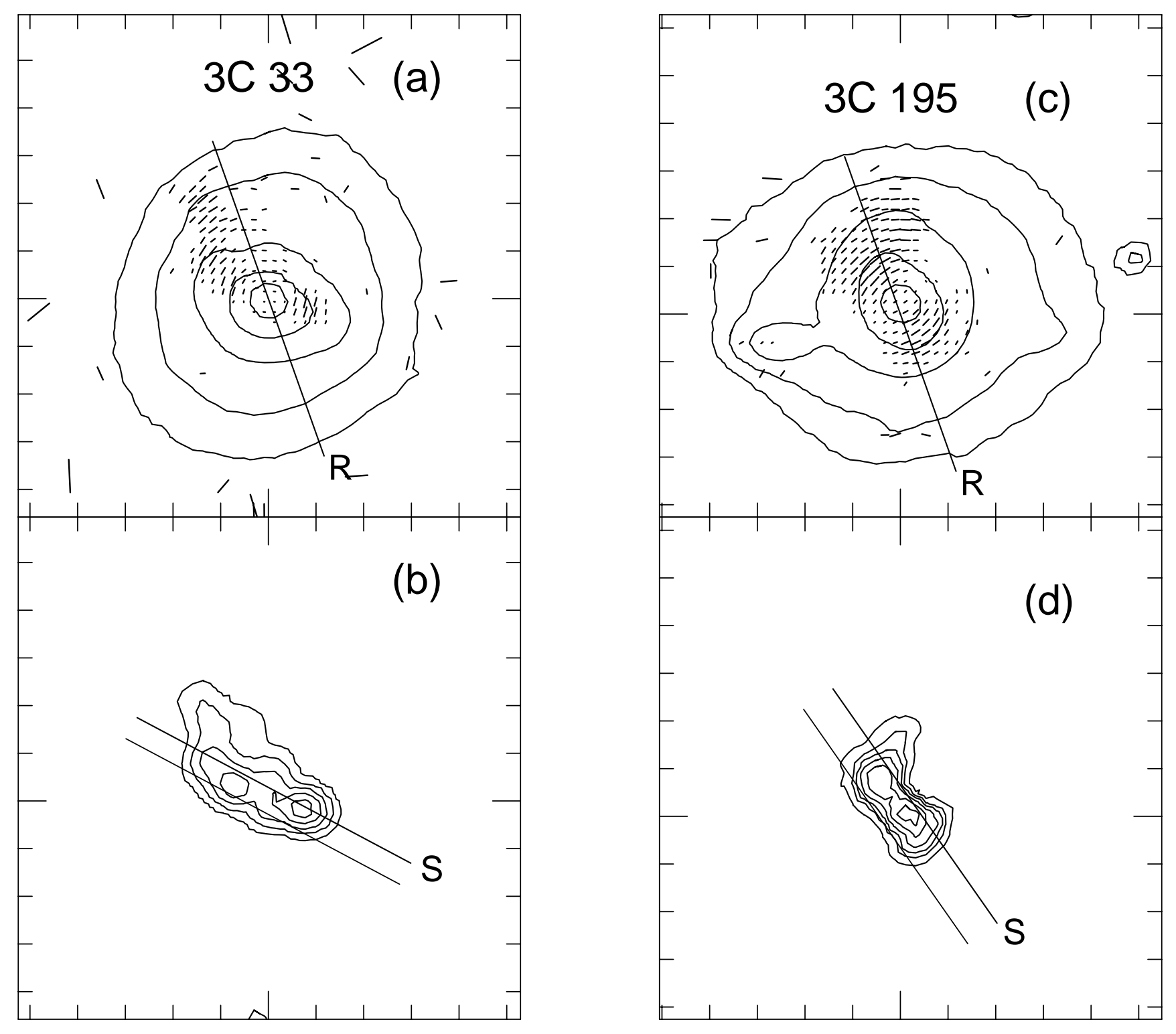

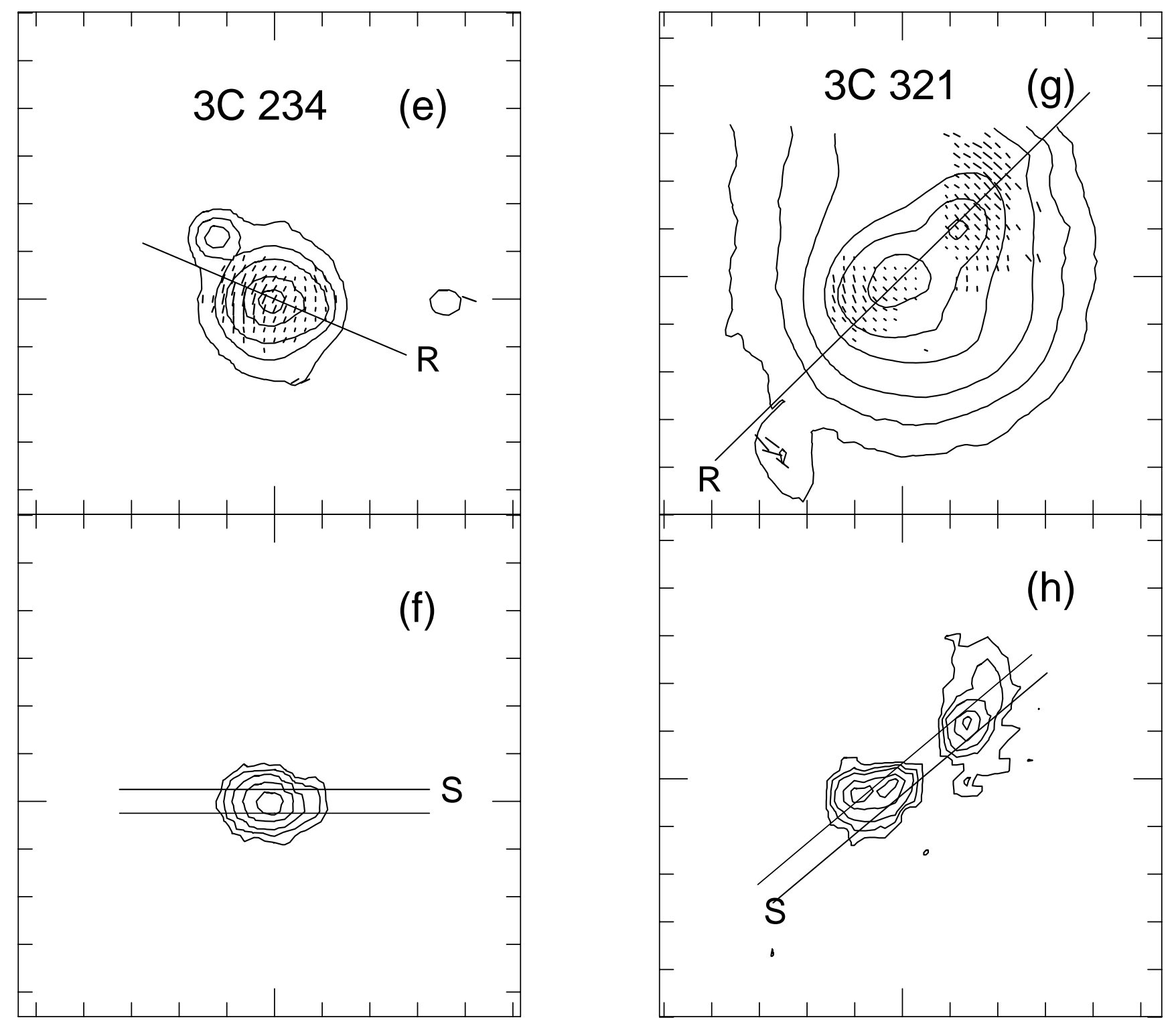


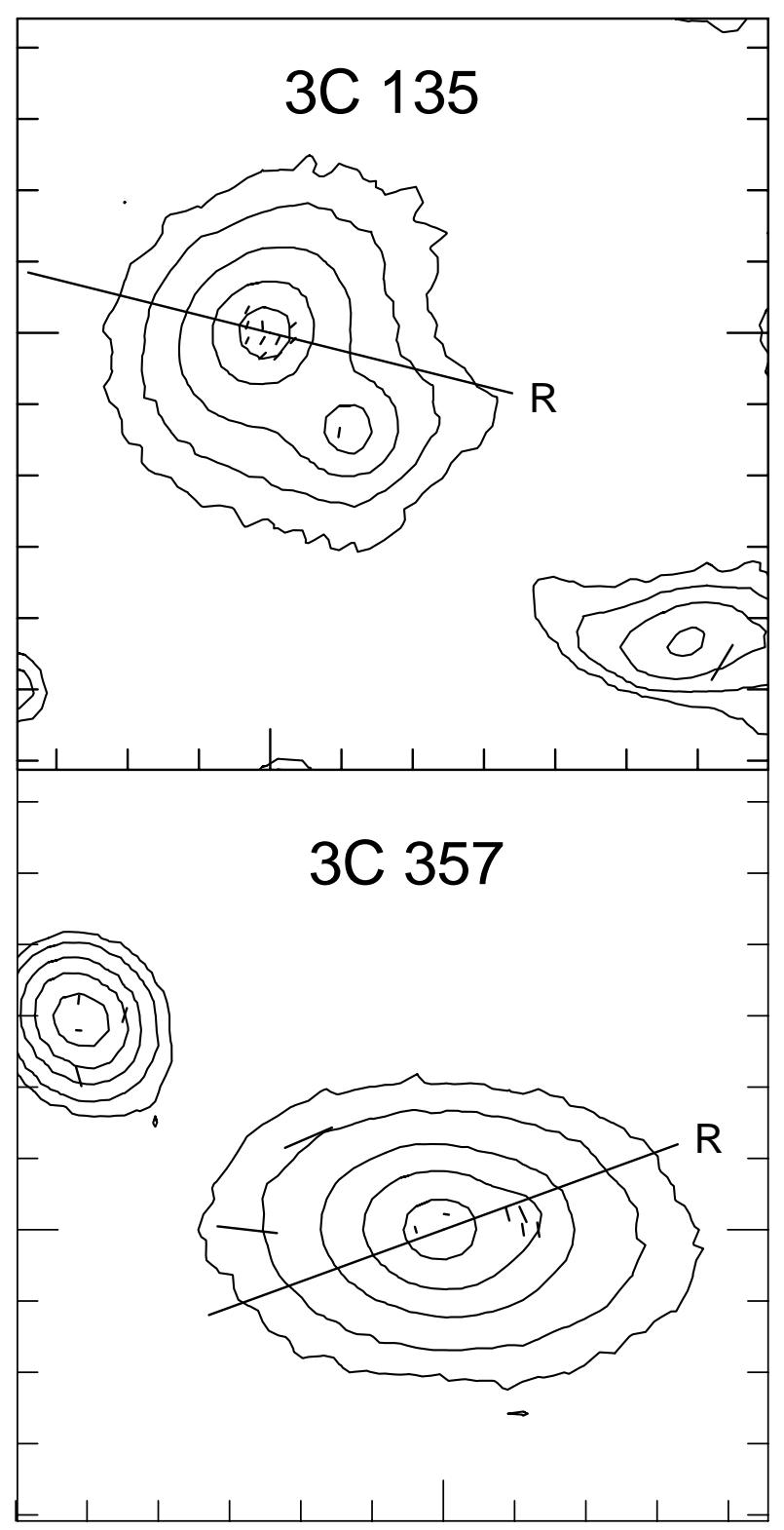




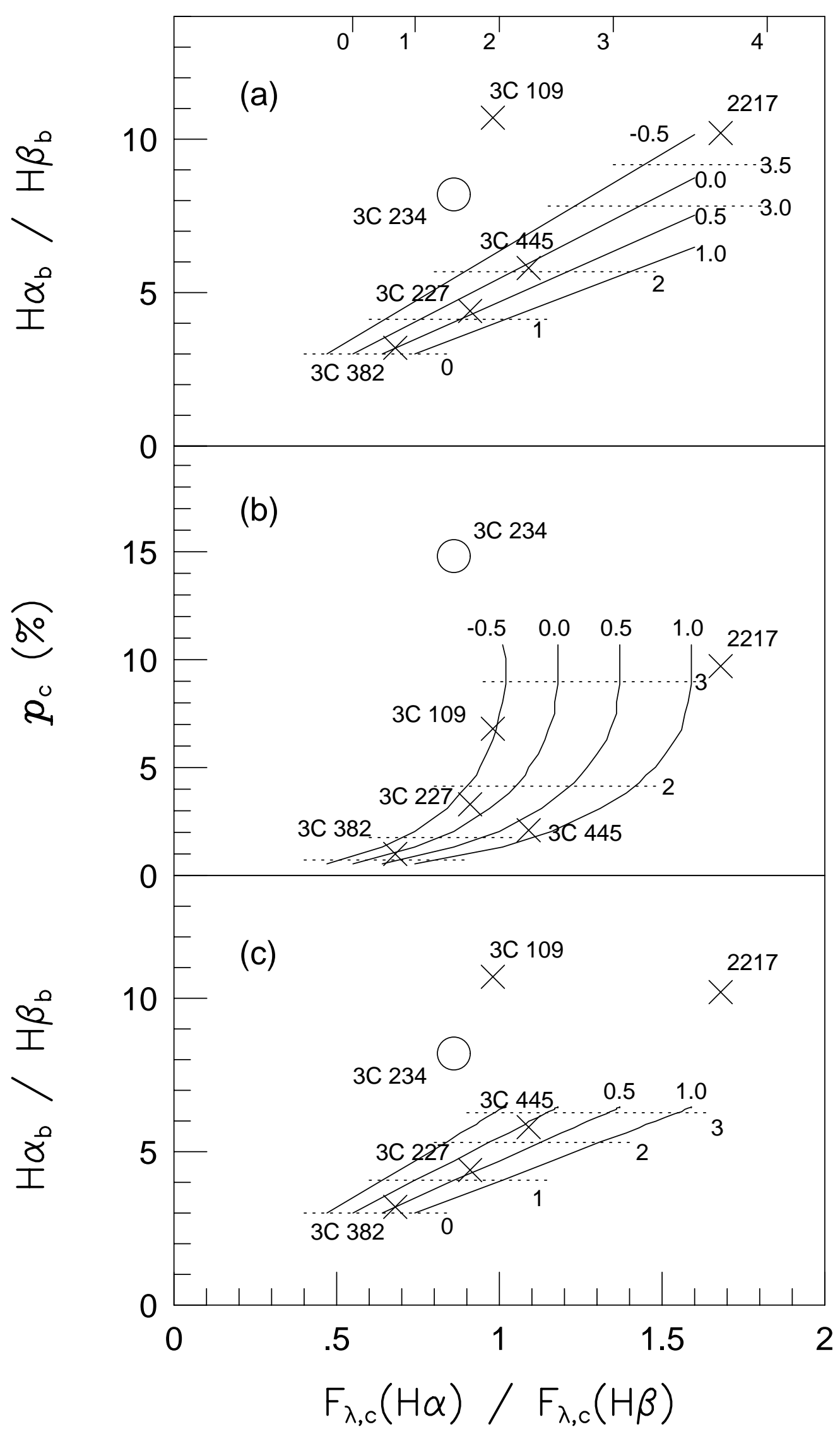




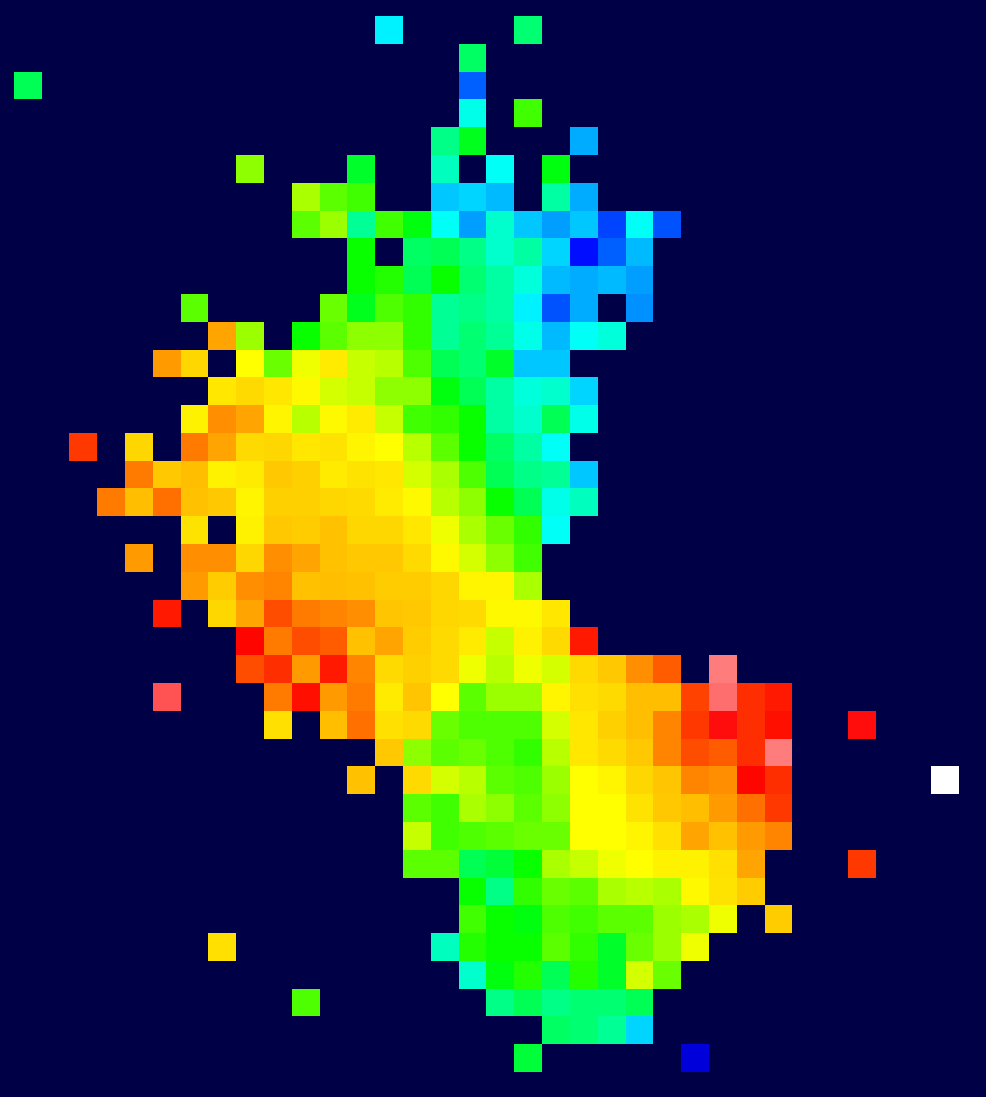




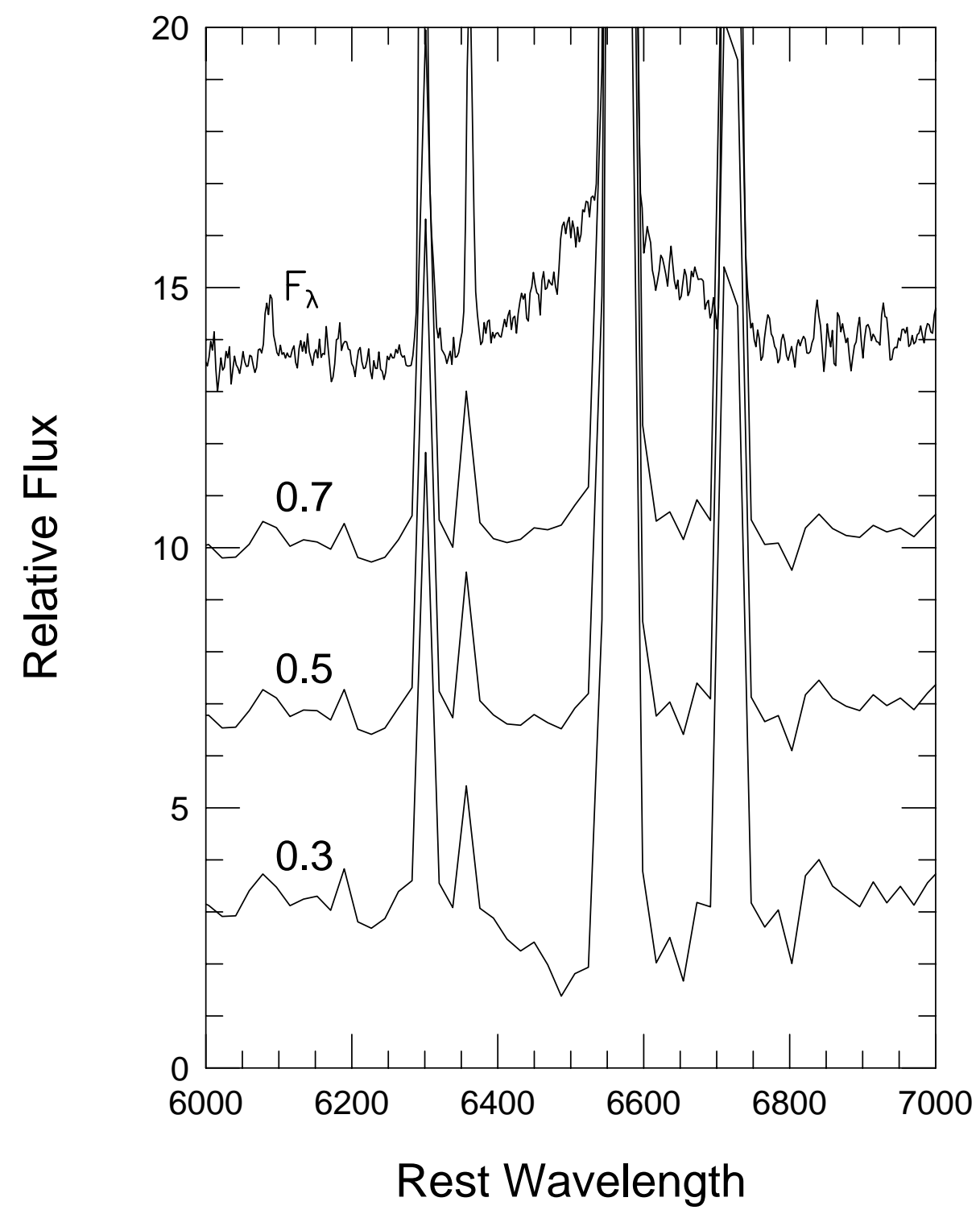




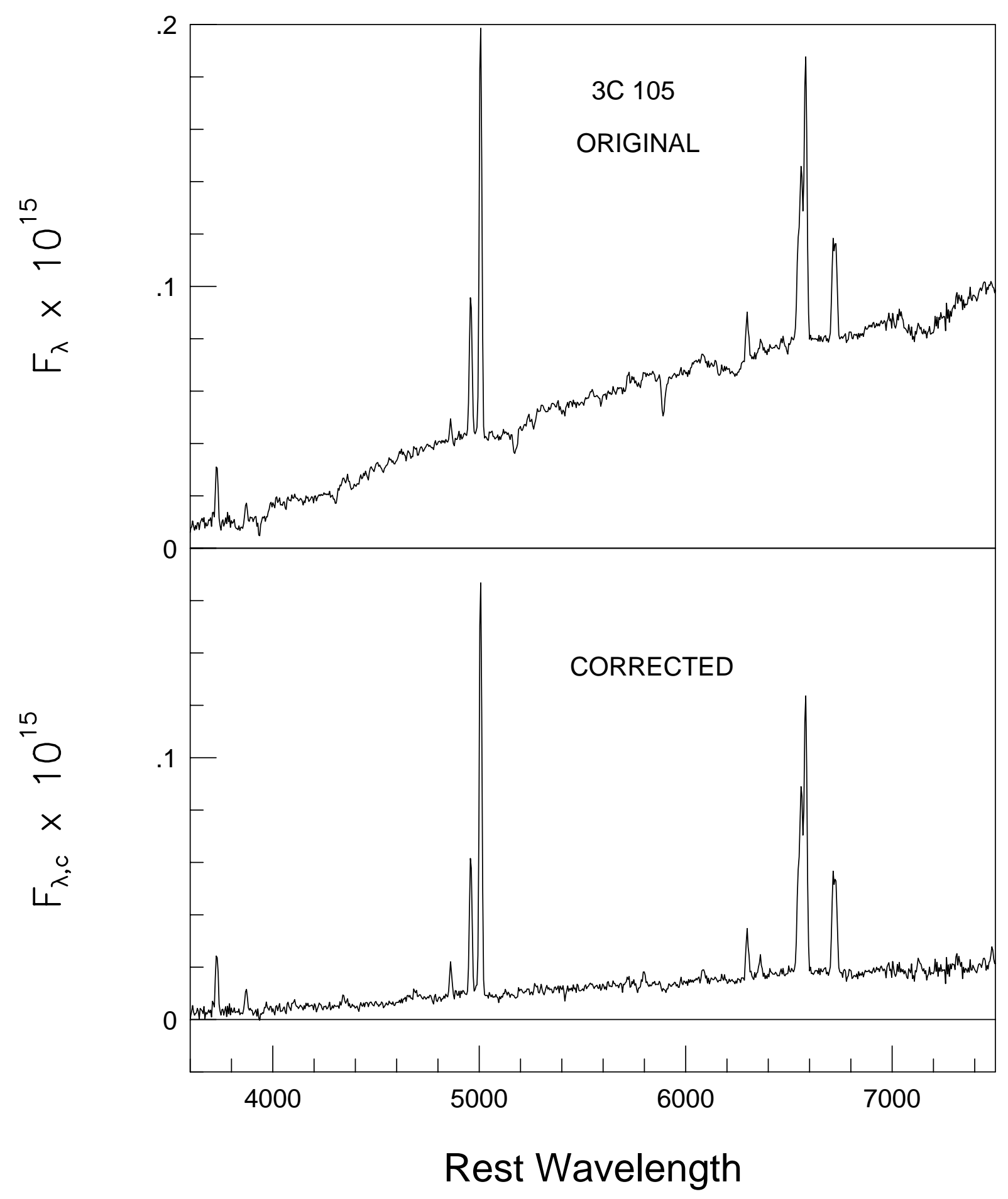




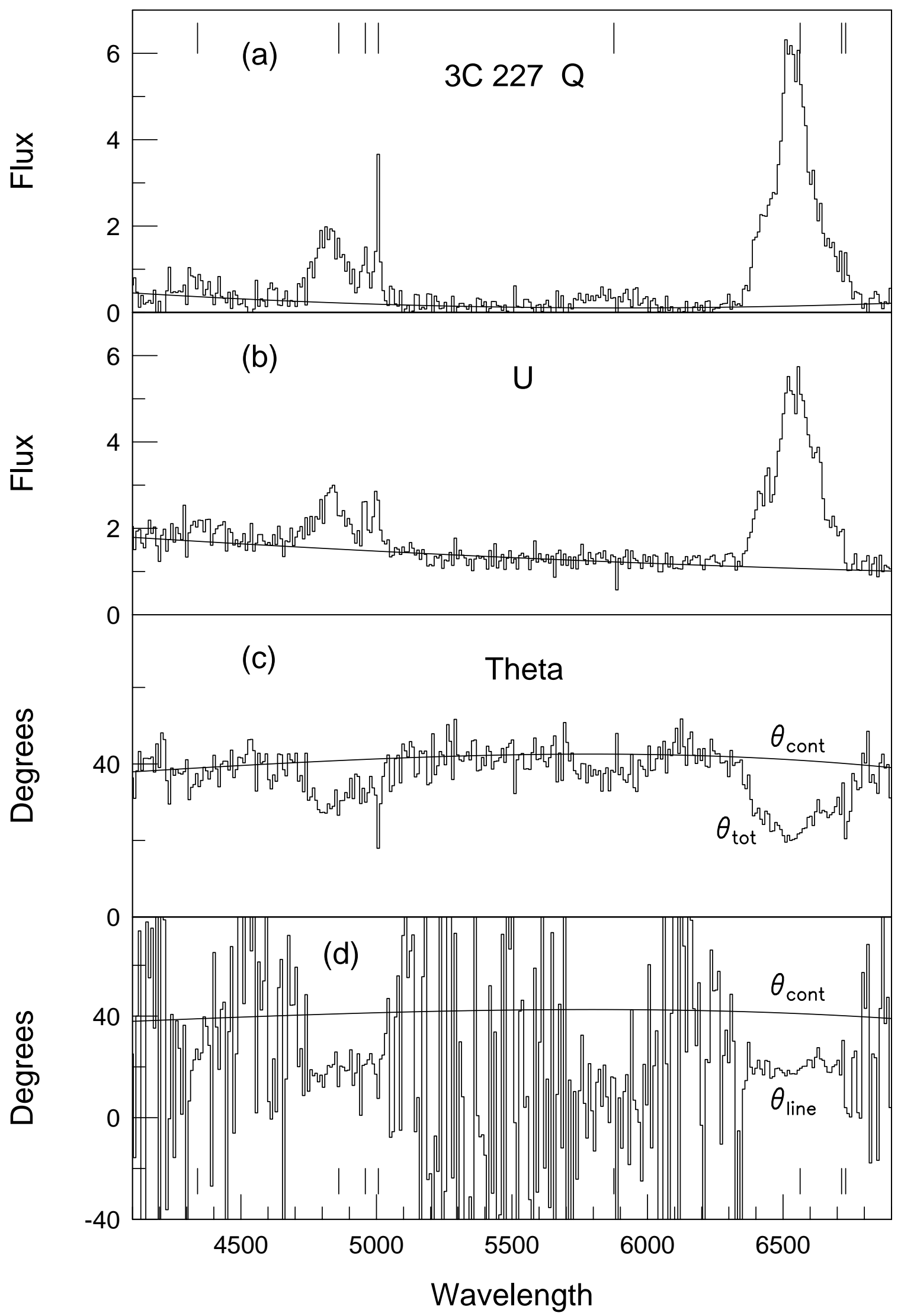




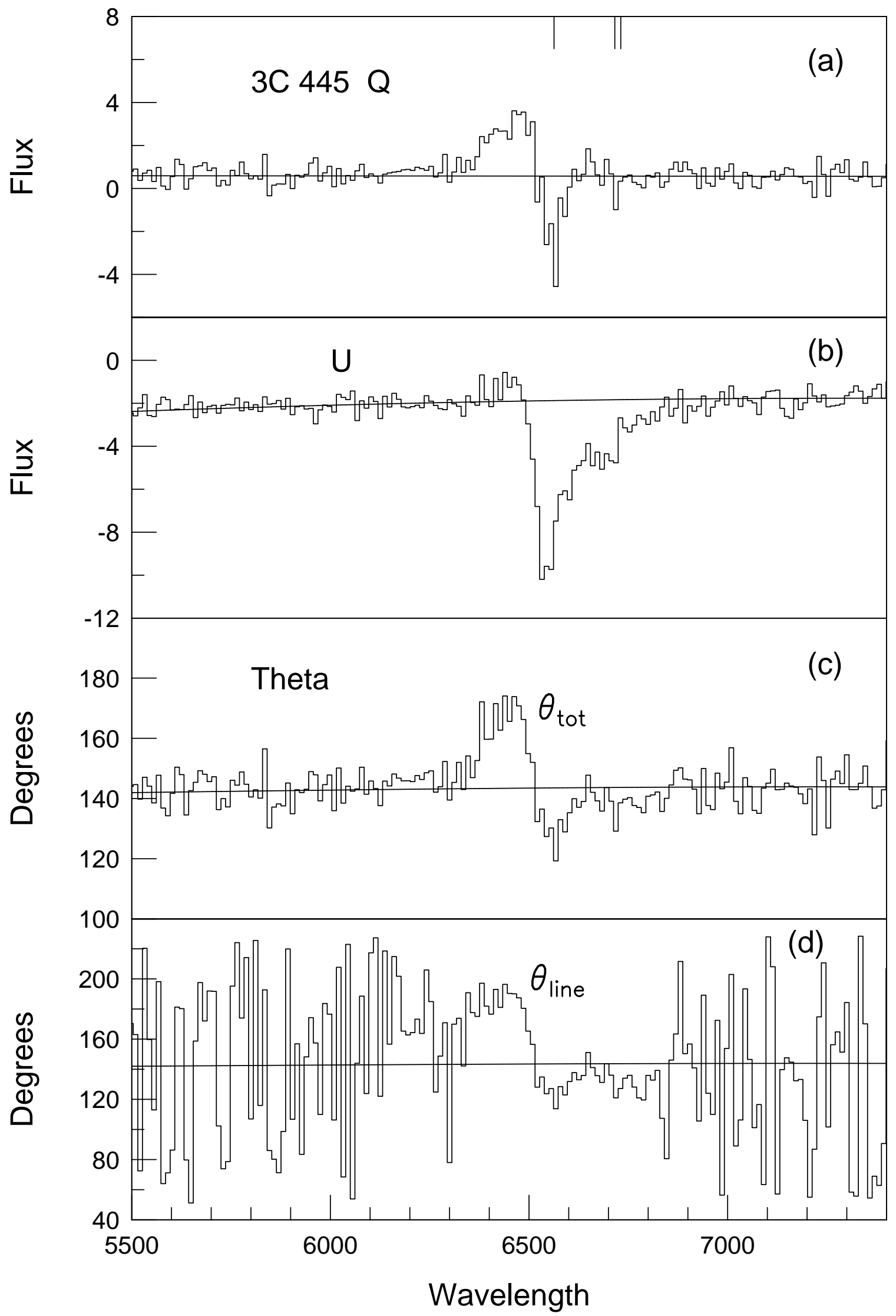

